\title{
Beam dynamics aspects of crab cavities in the CERN Large Hadron Collider
}

\author{
Yi-Peng Sun (孙一鹏), ${ }^{*}$ Ralph Assmann, Javier Barranco, Rogelio Tomás, Thomas Weiler, and Frank Zimmermann \\ European Organization for Nuclear Research (CERN), Geneva, Switzerland \\ Rama Calaga \\ BNL, Upton, New York 11973, USA
}

Akio Morita (森田 昭夫)

High Energy Accelerator Research Organization, KEK, 1-1 Oho, Tsukuba, Ibaraki 305-0801, Japan

(Received 19 May 2009; published 14 October 2009)

\begin{abstract}
Modern colliders bring into collision a large number of bunches to achieve a high luminosity. The longrange beam-beam effects arising from parasitic encounters at such colliders are mitigated by introducing a crossing angle. Under these conditions, crab cavities (CC) can be used to restore effective head-on collisions and thereby to increase the geometric luminosity. Such crab cavities have been proposed for both linear and circular colliders. The crab cavities are rf cavities operated in a transverse dipole mode, which imparts on the beam particles a transverse kick that varies with the longitudinal position along the bunch. The use of crab cavities in the Large Hadron Collider (LHC) may not only raise the luminosity, but it could also complicate the beam dynamics, e.g., crab cavities might not only cancel synchrobetatron resonances excited by the crossing angle but they could also excite new ones, they could reduce the dynamic aperture for off-momentum particles, they could influence the aperture and orbit, also degrade the collimation cleaning efficiency, and so on. In this paper, we explore the principal feasibility of LHC crab cavities from a beam dynamics point of view. The implications of the crab cavities for the LHC optics, analytical and numerical luminosity studies, dynamic aperture, aperture and beta beating, emittance growth, beam-beam tune shift, long-range collisions, and synchrobetatron resonances, crab dispersion, and collimation efficiency will be discussed.
\end{abstract}

DOI: 10.1103/PhysRevSTAB.12.101002

PACS numbers: 29.20.db

\section{INTRODUCTION}

The Large Hadron Collider (LHC) has a design luminosity of $10^{34} \mathrm{~cm}^{-2} \mathrm{~s}^{-1}$ at the two high-luminosity protonproton experiments ATLAS (located at interaction point 1 , or "IP1") and CMS (located at IP5), with a center-of-mass energy of $14 \mathrm{TeV}$ [1]. Studies aimed to further raise the LHC luminosity have been carried out since 2001, from 2004 onwards jointly by the European CARE-HHH network and by US-LARP. These studies developed a roadmap for increasing the luminosity of LHC by a factor of 10 above its design value, to $10^{35} \mathrm{~cm}^{-2} \mathrm{~s}^{-1}$ in the second half of the next decade. Recently, three upgrade scenarios have crystallized from various considerations. These three scenarios are called the early-separation (ES) scheme, the full crab crossing (FCC) scheme, and the large-Piwinski angle (LPA) scheme [2]. For the ES and FCC approaches, crab cavities are an essential ingredient of the upgrade [3].

Crab cavities could restore an effective head-on collision at the IP for both linear colliders [4] and circular colliders [5]. Namely, the crab cavities will render the effective Piwinski angle $\phi_{\text {Piw }}=\theta / 2 \cdot \sigma_{z} / \sigma_{x}{ }^{*}$ (with $\theta$ denoting the full crossing angle, $\sigma_{z}$ the rms bunch length, $\sigma_{x}{ }^{*}$ the

\footnotetext{
*Yipeng.SUN@cern.ch
}

rms horizontal beam size at IP) equal to zero, and minimize the geometric luminosity loss which otherwise arises from the crossing angle. By contrast, the LPA scheme relies on a nonzero Piwinski angle. It could only make use of crab cavities if the latter do not restore the beam-beam tune shift to the value obtained for a head-on collision, or if they increase the beam-beam tune-shift limit, or for the purpose of luminosity leveling.

In standard accelerator coordinates, the required horizontal kick from the crab cavity can be written as

$$
\Delta p_{x}=-\frac{\partial H_{\mathrm{crab}}}{\partial x}=-\frac{q V}{P_{s}} \cdot \sin \left(\phi_{s}+\frac{\omega z}{c}\right),
$$

where $\Delta p_{x}$ denotes the horizontal kick.

The Hamiltonian to describe the thin crab cavity is obtained by integration with respect to $x$ (in the Lie algebra sense [6])

$$
H_{\text {crab }}=\frac{q V}{P_{s}} \cdot \sin \left(\phi_{s}+\frac{\omega z}{c}\right) \cdot x,
$$

where $H_{\text {crab }}$ denotes the Hamiltonian, $q$ the particle charge, $V$ the voltage of the crab cavity, $P_{s}$ the particle energy, $\phi_{s}$ the synchronous phase of the crab-cavity rf wave, $\omega$ the angular $r f$ frequency of the crab cavity, $z$ the longitudinal 
coordinate of the particle with respect to the bunch center, $c$ the velocity of light, and $x$ the horizontal coordinate.

Because of symplecticity, the crab cavity also introduces an $x$-dependent longitudinal kick,

$$
\Delta p_{z}=-\frac{\partial H_{\text {crab }}}{\partial z}=-\frac{q V}{P_{s}} \cdot \cos \left(\phi_{s}+\frac{\omega z}{c}\right) \cdot \frac{\omega}{c} \cdot x .
$$

\section{LOCAL AND GLOBAL SCHEME}

In circular colliders, crab cavities may be configured according to either one of two schemes, namely as local or global crab cavities. In the local scheme, which corresponds to the original classical proposals of Palmer [4], Oide and Yokoya [5], a pair of crab cavities is placed at both sides of one IP, with the phase advance between crab cavity and IP optimized to be $\pi / 2$ in the crossing plane, as shown in Fig. 1 (left) and Fig. 2 (left); in that case, for each value of $z$ the crab cavities act like a local bump and the closed orbit in the other parts of the ring is not affected. The voltage needed for the first crab cavity (to rotate the bunch so as to be head-on at the interaction point) and for the second crab cavity (to rotate the bunch back) can be calculated using the formulas (4) and (5) below [7]. The two beams are crabbed at the specified IP as shown in Fig. 2 (left).

The crab-cavity voltage required on the two sides of the IP is

$$
\begin{gathered}
V_{1}=\frac{c^{2} \cdot p_{s} \cdot \tan \left(\frac{\theta}{2}\right)}{q \cdot \omega \cdot \sqrt{\beta^{*} \cdot \beta_{\text {crab }}} \cdot \sin \left(\Delta \varphi_{0}\right)}, \\
V_{2}=-R_{22} \cdot V_{1},
\end{gathered}
$$

where $V_{1}$ denotes the voltage of the first local crab cavity, $V_{2}$ the voltage of the second local crab cavity, $c$ the velocity of light, $p_{s}$ the particle momentum, $\theta$ the full crossing angle, $q$ the particle charge, $\omega$ the angular frequency of the crab cavity, $\beta^{*}$ the beta function at the interaction point,
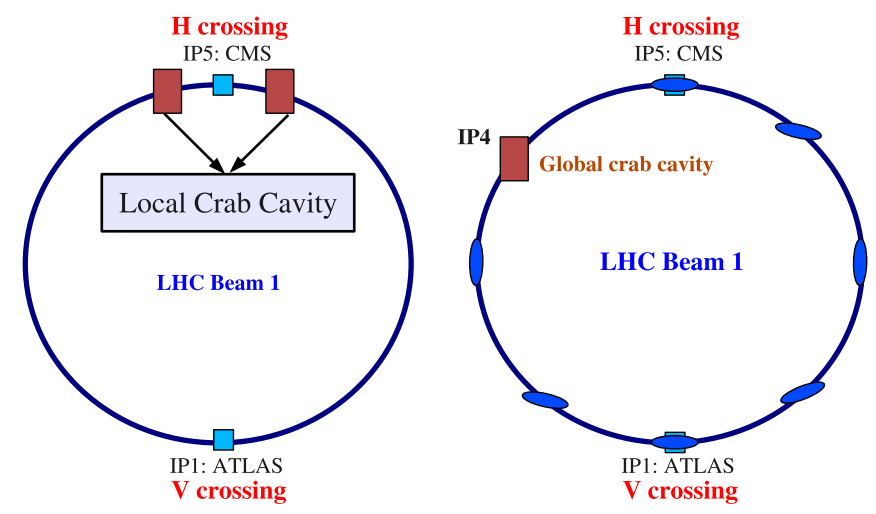

FIG. 1. (Color) Two local crab cavities in interaction region 5 (IR5) for LHC beam 1 which illustrates the local scheme (left) and one global crab cavity in IR4 for LHC beam 1 which illustrates the global scheme (right) (single global scenario).

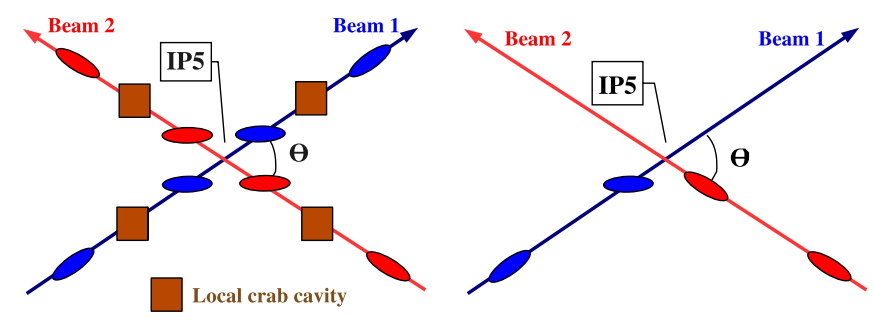

FIG. 2. (Color) Schematic for two crabbed beams at IP5, local scheme (with $\theta$ denoting the full crossing angle) (left); schematic of only one crabbed beam at LHC IP5 with a single global crab cavity, for beam 1 only (right).

and $\Delta \varphi_{0}$ the phase advance between the crab-cavity location and the IP. Lastly, $R_{22}$ signifies the $(2,2)$ element of the optical transport matrix from the first crab cavity to the second crab cavity, and the $R_{12}$ between the two cavities should be zero, $R_{12}=0$.

For the global scheme as sketched in Fig. 1 (right), the crab cavity is located somewhere around the ring, at a place which satisfies certain phase advance requirements [which is, $\left|\cos \left(\Delta \varphi_{0}-\pi Q\right)\right|=1$ in formula (6)] which involve the total betatron tune in addition to the phase advance from crab cavity to the IP; in the global case, the $z$-dependent closed orbit will be changed all around the ring with crab cavity active, and the crab-cavity voltage for the global scheme can be calculated from [7]:

$$
V=\frac{c^{2} \cdot p_{s} \cdot \tan \left(\frac{\theta}{2}\right)}{q \cdot \omega \cdot \sqrt{\beta^{*} \cdot \beta_{\text {crab }}}} \cdot\left|\frac{2 \sin (\pi Q)}{\cos \left(\Delta \varphi_{0}-\pi Q\right)}\right|,
$$

where $V$ denotes the voltage of the global crab cavity (GCC), $Q$ the betatron tune of the storage ring, and other parameters are the same as introduced before. The bunch will be tilted and the tilt will exhibit some kind of oscillation all around the ring such that at the interaction point the collision will be effectively head on. A global scheme has been implemented at KEKB [8].

\section{OPTICS AND SCENARIOS}

Crab cavities could be introduced for different phases of the LHC upgrade, for example, phase I with one global cavity for a feasibility test, and phase II for the final implementation using a local scheme. In this paper, we study the crab-cavity beam dynamics issues for two LHC optics, the nominal LHC collision optics and the so-called "lowbetamax" upgrade optics [9] (collision). The relevant parameters of these two optics are listed in Table I. As already discussed, in circular colliders crab cavities may be configured according to either local or global crab schemes, while the local scheme is the most ideal case for crab crossing. In the following, we will study two different crab crossing scenarios: (1) four local crab cavities at LHC IR5, two for LHC beam 1, and two for LHC beam 2 (both beams crabbed at IP5); (2) only one global crab cavity at IR4, to crab LHC beam 1 at IP5. 
TABLE I. Relevant parameters of the two LHC optics under study.

\begin{tabular}{lccc}
\hline \hline Parameter & Symbol & Nominal LHC optics & lowbetamax optics \\
\hline Protons per bunch & $N_{b}\left[10^{11}\right]$ & 1.15 & 1.15 \\
Number of bunches & $n$ & 2808 & 2808 \\
rms bunch length & $\sigma_{z}[\mathrm{~cm}]$ & 7.55 & 7.55 \\
rms energy spread & $\sigma_{e}\left[10^{-4}\right]$ & 1.1 & 1.1 \\
Beta function at IP1 \& IP5 & $\beta^{*}[\mathrm{~m}]$ & 0.55 & 0.25 \\
Emittance & $\epsilon\left[10^{-6} \mathrm{mrad}\right]$ & 3.75 & 3.75 \\
Full crossing angle & $\theta[\mu \mathrm{rad}]$ & 285 & 381 \\
\hline \hline
\end{tabular}

\section{A. Local scenario}

First we discuss the possible installation of the local crab cavities in LHC IR5 (local scenario), to benefit the protonproton collision at IP5 (for IP1 the procedure is almost the same). For the nominal LHC collision optics, the location of the first local crab cavity is selected to be upstream of IP5, outside D2 (the second dipole from IP5), and between Q4 and Q5 (proceeding from IP5 to either side, the main quadrupoles are named as Q1, Q2, Q3, Q4, and Q5 ...). We selected this location as here beams 1 and 2 have reached their full arc separation and the phase advance to IP5 is

TABLE II. Local crab-cavity voltage for LHC nominal collision optics and lowbetamax collision optics.

\begin{tabular}{lcc}
\hline \hline & LHC nominal collision & lowbetamax \\
\hline$V_{\mathrm{CC} 1}[\mathrm{MV}]$ & 3.5 & 4.9 \\
$V_{\mathrm{CC} 2}[\mathrm{MV}]$ & 0.7 & 3.1 \\
$\beta_{\mathrm{CC} 1}[\mathrm{~m}]$ & 1541 & 2616 \\
$\beta_{\mathrm{CC} 2}[\mathrm{~m}]$ & 665 & 1023 \\
$\varphi_{\mathrm{IP}}-\varphi_{\mathrm{CC} 1}[2 \pi]$ & 0.259 & 0.254 \\
$\varphi_{\mathrm{CC} 2}-\varphi_{\mathrm{IP}}[2 \pi]$ & 0.259 & 0.254 \\
$R_{22}$ & -0.2 & -0.63 \\
\hline \hline
\end{tabular}

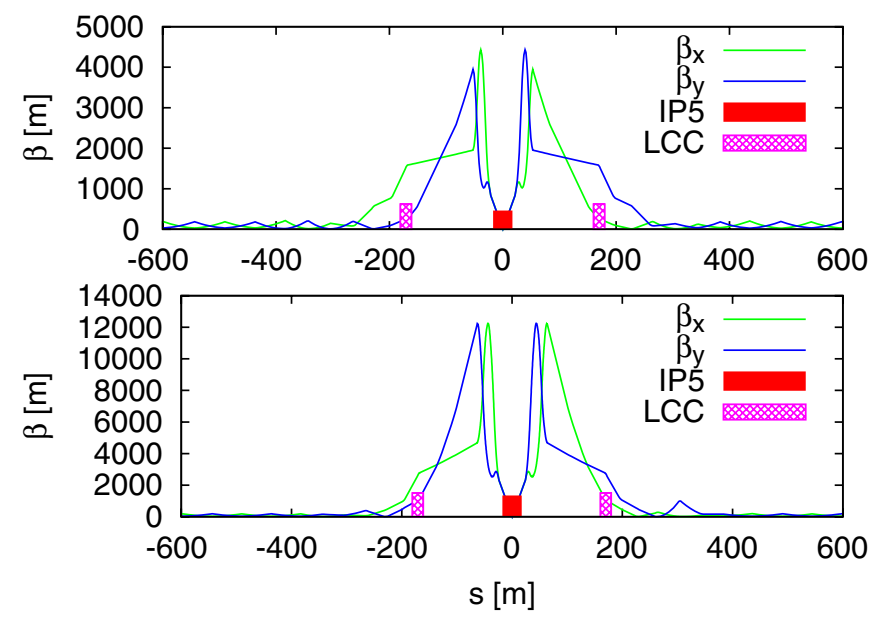

FIG. 3. (Color) Optics parameters for the local crab scheme in LHC IR5: nominal LHC collision optics (top) and lowbetamax collision optics (bottom). IP5 is at $s=0$. near $\pi / 2$. The longitudinal distance between the selected position and IP5 is around 170-171 $\mathrm{m}$, and the horizontal phase advance between IP5 and the first local crab cavity is around 0.259 (in units of $2 \pi$ ). The second crab cavity is placed symmetrically (in phase) at the other side of the IP5. For the lowbetamax collision optics, the convention of the local crab cavities is almost the same [10]. The required crab voltage and associated $\beta$ function at the crab cavities are summarized in Table II. The required voltage may be provided by a pair of two-cell superconducting cavities. In Fig. 3 the optics parameters and the local crab cavity are plotted together at IR5, for the nominal LHC collision optics and the lowbetamax collision optics, respectively.

\section{B. Global scenario (single global)}

Because of the space constraints in the LHC tunnel on the two-beam separation, and other factors, it appears much more realistic that, in an initial, exploratory phase, only one global crab cavity ( $800 \mathrm{MHz})$ will be installed in IR4 (where the separation between the two beams is largest and cryogenics plus other needed infrastructure might be more easily available), to test the crab cavity in hadron colliders for the first time. In this scenario (single global), only a single global crab cavity will be used to obtain one horizontally crabbed beam at IP5. A schematic of the LHC layout with one global crab cavity is shown in Fig. 1 (right), while the collision case with a single crabbed beam (at IP5) is sketched in Fig. 2 (right).

In LHC IR4 there presently exists a free space of $5 \mathrm{~m}$ length for both beam 1 and beam 2, which is reserved for future $200-\mathrm{MHz}$ capture rf cavities, and just next to the LHC main rf systems (at IP4). This region is also favorable as a potential location of the global crab cavity because it is near to the existing cryogenic and other infrastructures of the main LHC rf systems. The phase advance for LHC beam 1 between this $200-\mathrm{MHz}$ capture rf location and IP5 is good, with $\cos (\Delta \varphi-\pi Q)=-0.9978$. The selected location for crab cavity is around $30 \mathrm{~m}$ upstream of IP4.

The $800-\mathrm{MHz}$ crab-cavity voltage is calculated to be 9.3 MV, which is a reasonable value for three two-cell cavities. The LHC optics in IR4 will be adjusted later if the voltage needs to be further decreased. A preliminary study indicates that the horizontal beta function at the crab cavity (CC) $\beta_{x, \mathrm{CC}}$ can be increased up to $3000 \mathrm{~m}$ while 
maintaining a matched optics for the nominal LHC at $7 \mathrm{TeV}$ [11]. This optics change will decrease the required crab-cavity voltage to about $2.3 \mathrm{MV}$, which is within the reach of existing superconducting rf technology for a twocell cavity. For the purpose of this study, the nominal LHC optics V6.501 is left unchanged, and the global crab cavity is installed at the reserved $200-\mathrm{MHz}$ rf location ("ADTKH" in LHC sequence V6.501), for beam 1.

For the lowbetamax collision optics, the installation procedure is similar [10], and the $800-\mathrm{MHz}$ crab-cavity voltage is calculated to be $25 \mathrm{MV}$ which requires that the $\beta$ function at the crab cavity $\left(\beta_{\mathrm{CC}}\right)$ should be increased a lot (again, the same preliminary study indicates that $3000 \mathrm{~m}$ is possible). For the lowbetamax the IR4 optics is essentially the same as the nominal one, and one would, therefore, expect roughly the same gain factor in the crab voltage in the case of the global crab-cavity scheme. The limits on the cavity voltage could also be overcome by changing the working point and going closer to the integer resonance. It has been demonstrated that the LHC optics is so flexible as to permit tune changes up to an integer in either direction and for the two transverse planes [12]. As the required global crab voltage scales with $\sin (\pi Q)$ [for constant $\cos \left(\Delta \varphi_{0}-\pi Q\right)$ ], for a fractional tune 0.05 , e.g., we can reduce the crab voltage by $\sin (0.05 \pi) / \sin (0.31 \pi)=0.19$, which translates to more than a factor 5 reduction in the required crab voltage. These two measures, applied together, can decrease the required crab voltage from 25 to 1.3 MV. We note that here we do not propose the routine operation with full intensity at tunes close to the integer resonance, but only a change of tune for the purpose of a dedicated experiment with a crab-cavity prototype in order to obtain a significant relative luminosity gain even at fairly low crab-cavity voltage. The global crab optics at LHC IR4 is shown in Fig. 4, for the nominal LHC collision

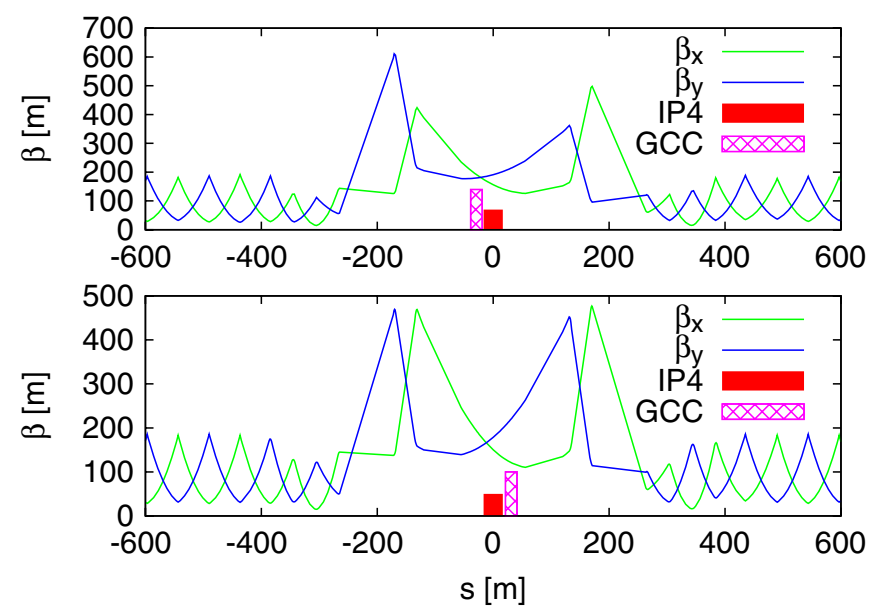

FIG. 4. (Color) Global crab optics at LHC IR4: nominal LHC collision optics (top) and lowbetamax collision optics (bottom). IP4 is at $s=0$.

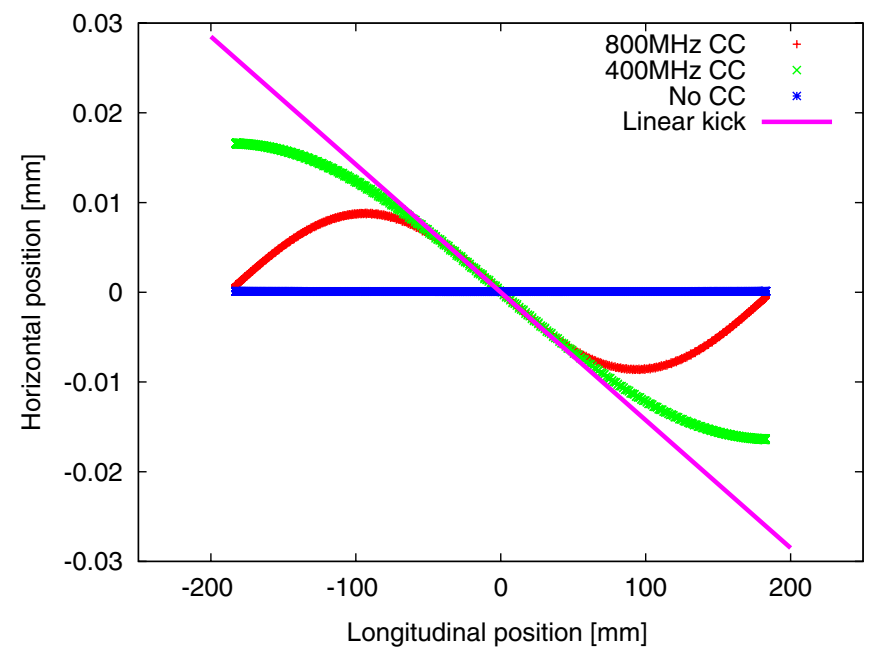

FIG. 5. (Color) Turn-by-turn $x-z$ correlation of an offmomentum particle $\left(2.5 \sigma_{p}\right)$ at IP5 with $400-\mathrm{MHz}$ and $800-$ $\mathrm{MHz}$ global crab cavity, for the nominal LHC optics (with rms bunch length $\sigma_{z}=75.5 \mathrm{~mm}$ ). The crab-cavity kick is linear within $1 \sigma_{z}$, which recovers most of the geometric luminosity expected without crossing angle.

optics and for the lowbetamax collision optics, respectively.

The simulated turn-by-turn longitudinal-horizontal correlation of a particle is shown in Fig. 5, with the $800-\mathrm{MHz}$ single global scenario (as well as for a $400-\mathrm{MHz}$ crabcavity case with twice the voltage) in comparison with an ideal totally linear crab-cavity rf. To get the simulated $x-z$ correlation shown, one particle with zero transverse and longitudinal offset, and $2.5 \sigma_{p}$ energy offset has been tracked for 1000 turns. In the simulation, one virtual beam-position monitor (BPM) has been placed at IP5 to record the position of this particle turn by turn. The crab cavity has a sinusoidal rf-wave curvature, so that its kick imparted on the beam is linear only over a limited time range. From Fig. 5, it can be inferred that for the $800-\mathrm{MHz}$ crab cavity and a nominal LHC proton bunch length $\sigma_{z}$ of $0.075 \mathrm{~m}$, the crab-cavity kick is linear only within about $1 \sigma_{z}$. It can also be seen that the linearity of the $400-\mathrm{MHz}$ crab cavity over the length of the proton bunch is much better than that of the $800-\mathrm{MHz}$ cavity. However, the cavity size will be much larger for the 400-MHz frequency, which makes it difficult to install such a cavity on the beam line in the LHC tunnel. $800 \mathrm{MHz}$ represents a compromise between linearity, crab voltage, and transverse cavity size. The ideally required $x-z$ correlation of the bunch at IP5 is also illustrated by the curve for the "linear kick" in Fig. 5.

\section{DYNAMIC APERTURE}

For LHC at $7 \mathrm{TeV}$ (top energy), to obtain a sufficiently linear motion of particles at a transverse amplitude of $6 \sigma$ [which is the half jaw opening of the primary collimators (TCPs)], the simulated dynamic aperture (maximum stable 
amplitude of motion) is required to be at least a factor of 2 larger, more than $12 \sigma$, without collisions. With collisions the dynamic aperture will be dominated by the long-range beam-beam effect, which will be discussed in Sec. XI. The dynamic aperture is determined by tracking particles with different initial coordinates using the SIXTRACK [13] code over 100000 turns, which corresponds to $1 / 400$ hour and is limited by the computing ability. The phase-space angle between the horizontal and vertical action variables $\left(J_{x}\right.$ and $J_{y}$ ) is defined to be $\phi=\operatorname{atan}\left(\sqrt{J_{y} / J_{x}}\right)$, and, for a complete dynamic-aperture simulation, the transverse launch angle $\phi$ is selected to be $15,30,45,60$, and 75 degrees, respectively [14].

Concerning optics imperfections, the measured magnetic errors are included (both normal and skew multipole coefficients) up to 30-pole for all dipoles and quadrupoles. Tune and chromaticity are corrected, and the corrections of the main dipole field errors by the $b_{3}, b_{4}$, and $b_{5}$ spoolpiece families are taken into account. The nonlinear magnetic errors are modeled for the main dipoles and quadrupoles, plus the insertion quadrupoles and cold D1 (D2) dipoles, and also the warm quadrupoles. The beam energy is $7 \mathrm{TeV}$ and the initial momentum offset is set to be 0.00027 (3/4 of the rf bucket; this corresponds to the standard convention for all LHC dynamic-aperture studies).

From the minimum dynamic aperture (nominal LHC) over 60 seeds of the nonlinear magnetic errors, which is shown in Fig. 6, we observe a maximum $1 \sigma$ degradation of the dynamic aperture compared to the nominal case without crab cavity. For the lowbetamax collision optics, the loss of dynamic aperture is $1 \sigma$ to $2 \sigma$, with the corresponding results shown in Fig. 7. In fact, the horizontal phase advance between the two local crab cavities, at the loca-

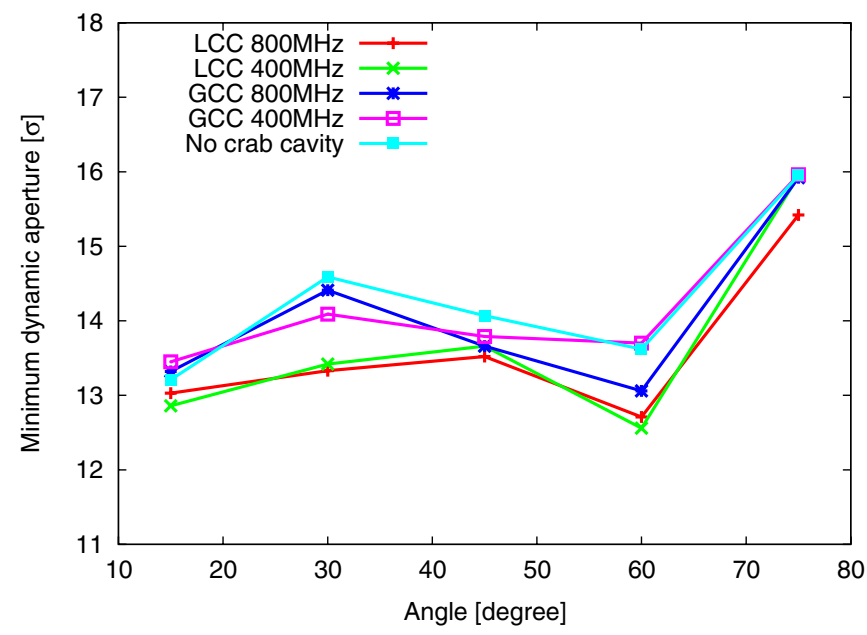

FIG. 6. (Color) Minimum dynamic aperture over 60 error seeds for nominal LHC optics with or without crab cavity; the maximum decrease of $1 \sigma$ due to crab cavity is acceptable. The dynamic-aperture tracking is performed for 100000 turns.

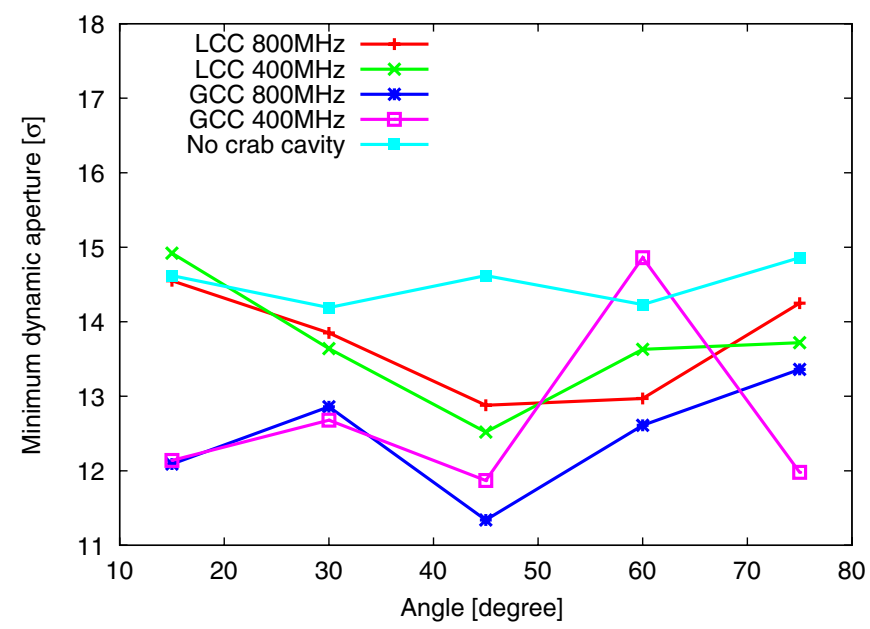

FIG. 7. (Color) Minimum dynamic aperture over 60 error seeds for lowbetamax collision optics with or without crab cavity; the maximum decrease of 1 or $2 \sigma$ due to crab cavity is acceptable. The dynamic-aperture tracking is performed for 100000 turns.

tions considered, is 0.518 (in units of $2 \pi$ ) for the nominal LHC, while it is 0.508 (in units of $2 \pi$ ) for the lowbetamax collision optics. The larger difference from the ideal value of 0.5 results in a smaller dynamic aperture for the local crab scheme at the nominal LHC, due to the imperfect $\pi$ bump and the resulting leakage of the crab orbit all around the machine. For this case, the dynamic aperture could indeed be fully recovered by optimizing the phase advance (in the crossing plane) between the two local crab cavities to be much closer to 0.5 (in units of $2 \pi$ ), as is demonstrated by simulations in Fig. 8. Realization of this optimum phase distance is not straightforward, however, since the opti-

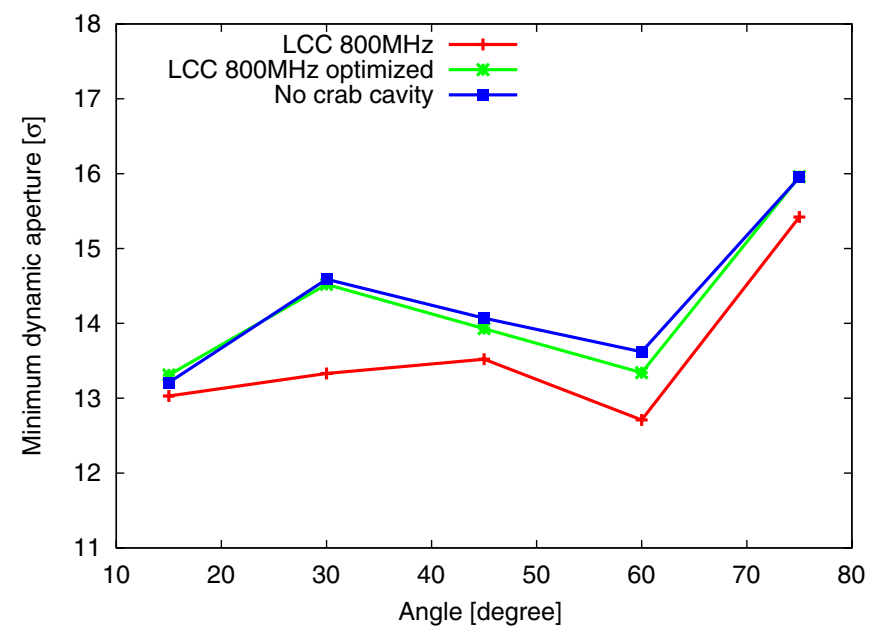

FIG. 8. (Color) Minimum dynamic aperture over 60 error seeds for nominal LHC optics, without crab cavity (blue); with two local $800-\mathrm{MHz}$ crab cavities and horizontal phase advance 0.518 (in units of $2 \pi$ ) between them (red); with two local $800-\mathrm{MHz}$ crab cavities and optimized horizontal phase advance 0.5 (in units of $2 \pi$ ) between them (green). 
mum location of the second crab cavity is occupied by other ring elements in the present optics. In addition, the phase advance from the first local crab cavity to the second is modified by the (head-on) beam-beam collision, as we will discuss in Sec. XI. Regardless, a simulated dynamic aperture above $12 \sigma$ is considered to be more than sufficient for LHC, given the fact that the primary collimator has a half opening of $6 \sigma$. For the global crab scheme the unavoidable additional modulated orbit offset around the entire ring, introduced by the crab cavity, causes the small loss in the dynamic aperture. It is still being studied how to compensate that effect.

\section{APERTURE AND BETA BEATING}

LHC will operate with superconducting magnets and superconducting rf cavities. Considering the huge beam power, a tiny loss of beam particles will result in a quench and may even damage the accelerator. The LHC collimation system, which is designed to protect the superconducting ring, requires a minimum mechanical aperture of $10 \sigma$ in terms of the rms beam sizes. To meet such a tight aperture limit, the optics and mechanical imperfections should be very well controlled.

The bunch is tilted everywhere in the ring and the closed orbit is modulated by the longitudinal position inside the bunch for the global crab scheme. To investigate the additional aperture budget needed to accommodate the tilted bunch in the global crab scheme, for a particle at the specified longitudinal location in a bunch, the effect of the crab cavity can be modeled by adding a horizontal corrector (with $z$-dependent strength) at the location of the crab cavity, according to

$$
\Delta p_{1}=-\frac{q V}{p_{s}} \sin \left(\frac{\omega z}{c}\right)
$$

where $\Delta p_{1}$ denotes the deflection angle of the corrector, $q$ the particle charge, $p_{s}$ the particle energy, and $z$ the longitudinal coordinate of the particle with respect to the bunch center.

By applying formula (7) to the nominal LHC collision optics and to the lowbetamax optics, at the same time considering the effect of one $800-\mathrm{MHz}$ or $400-\mathrm{MHz}$ global crab cavity, we get the horizontal orbit change due to the crab cavity, and compare it with the off-momentum orbit. The results are shown in Figs. 9 and 10, for the nominal LHC collision optics and the lowbetamax optics, respectively. This compares the size of the offset between the offmomentum beat and the additional offset (if any) from the crab cavity. We observe that the global scheme for nominal LHC requires an additional $0.5 \sigma$ aperture while the lowbetamax optics requires more, about $1 \sigma$.

The off-momentum beta beating is very important for the collimation system and the safe operation of the superconducting ring, as it can cause additional beam loss and introduce the overlap of the primary and secondary colli-

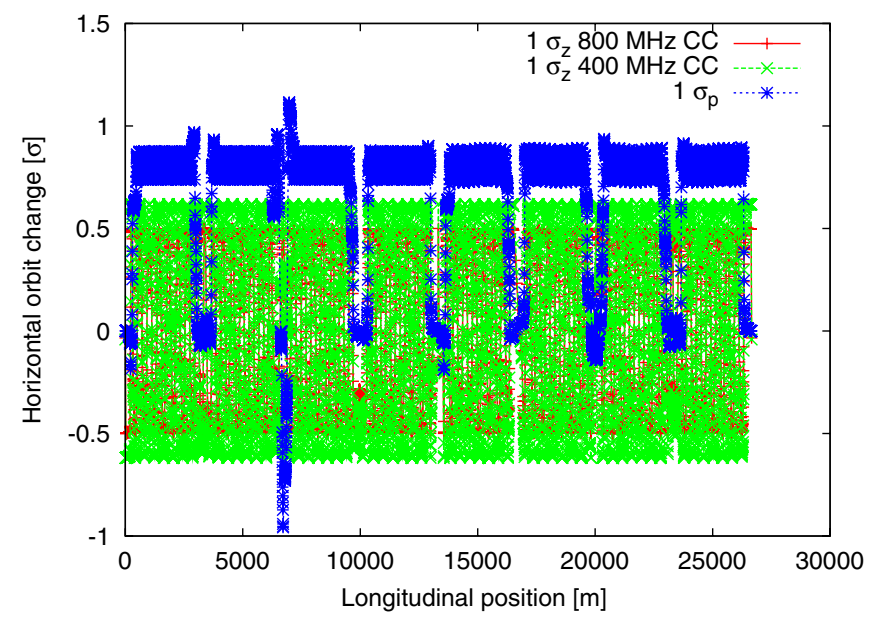

FIG. 9. (Color) Comparison of horizontal orbit change of a particle with $1 \sigma_{z}$ offset for $800-\mathrm{MHz}$ global crab-cavity case (red); of a particle with $1 \sigma_{z}$ offset for $400-\mathrm{MHz}$ global crabcavity case (green); and for a $1 \sigma_{p}$ off-momentum particle (blue), in the nominal LHC collision optics. The orbit change due to the crab cavity is comparable to, or smaller than, the dispersive orbit shift.

mators. The maximum beta-beating tolerance for LHC of $20 \%$ is limited by the magnet imperfections and the mechanical aperture of the hardware. The phase advances are optimized for small off-momentum beta beating in the region between IP1 to IP5 for the nominal LHC optics under consideration. Crab cavities introduce another kind of beta beating, which depends on the longitudinal position inside the bunch ( $z$ dependent). The effect of the crab cavity is again modeled by replacing the crab cavity with

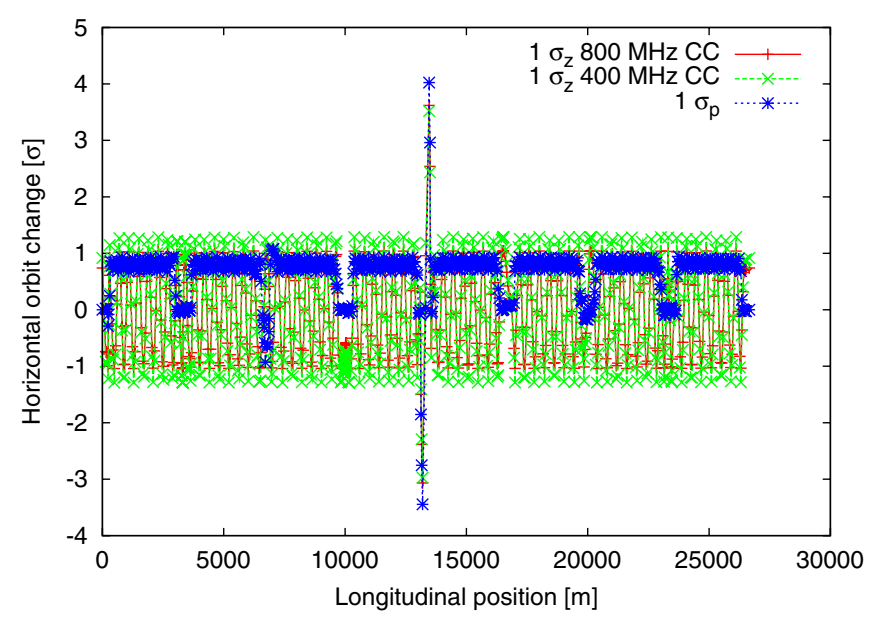

FIG. 10. (Color) Comparison of horizontal orbit change of a particle with $1 \sigma_{z}$ offset for $800-\mathrm{MHz}$ global crab-cavity case (red); of a particle with $1 \sigma_{z}$ offset for $400-\mathrm{MHz}$ global crabcavity case (green); and for a $1 \sigma_{p}$ off-momentum particle (blue), in the lowbetamax collision optics. The orbit change due to the crab cavity is comparable to, or smaller than, the dispersive orbit shift. 


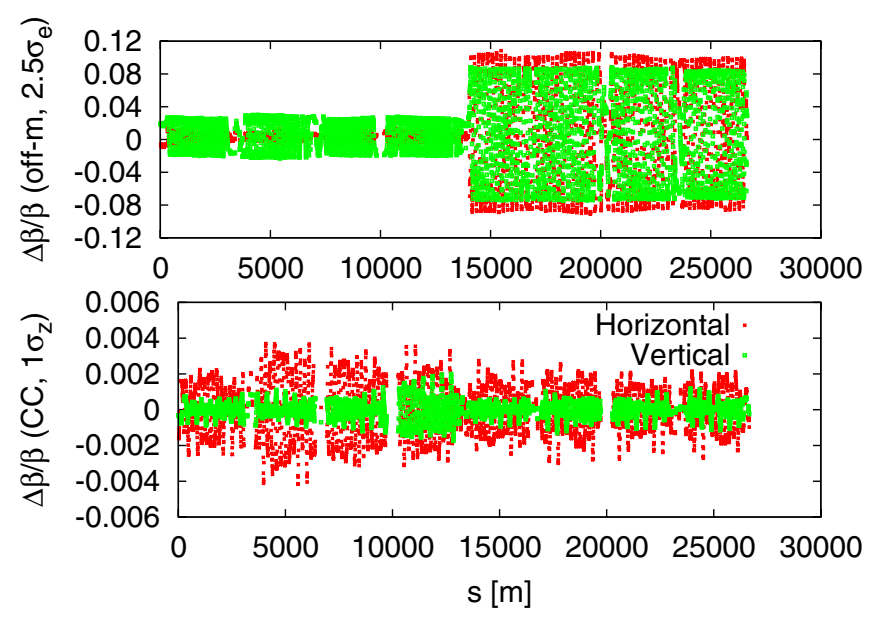

FIG. 11. (Color) Off-momentum beta beating for the nominal LHC optics with a relative momentum offset of 0.00027 (top); $z$-dependent beta beating due to the global crab cavity (bottom), which is much smaller than the off-momentum beta beating.

a horizontal dipole corrector (with $z$-dependent strength) in the sequence, according to formula (7).

With this method we can study this new kind of beta beating for a particle with longitudinal offset $z=1 \sigma_{z}$. From formula (7) we observe that the beta beating from crab cavity scales with $\sin (\omega z / c)$, while $z=1 \sigma_{z}$ is about the worst case. For the nominal LHC collision optics, the additional beta beating caused by the $800-\mathrm{MHz}$ global crab cavity is comparatively small with respect to the existing $\delta$-dependent off-momentum beta beating (at $3 / 4$ of the rf bucket height), as is shown in Fig. 11. For the lowbetamax optics, we observe a similar result and the maximum beta beating caused by the crab cavity lies within $\pm 0.6 \%$, as shown in Fig. 12.

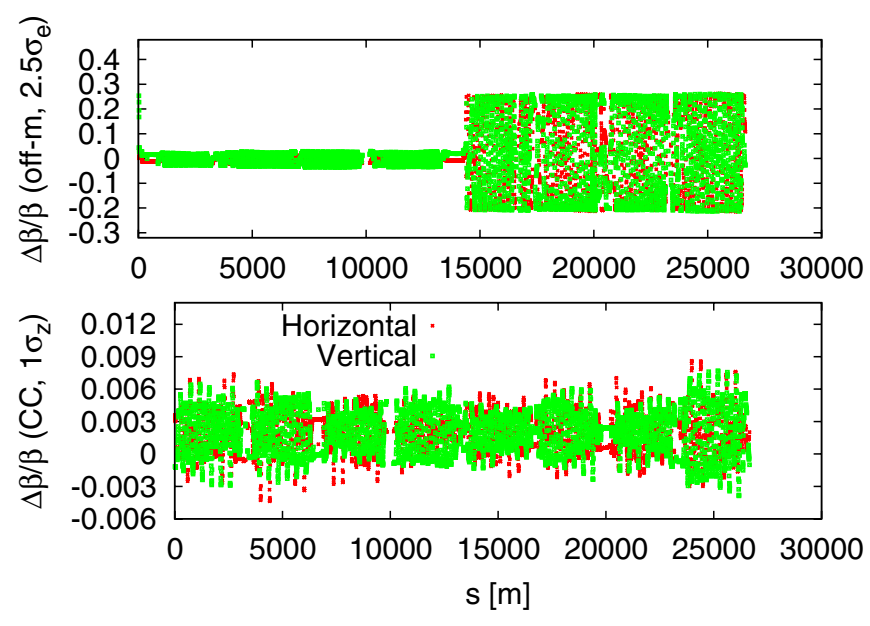

FIG. 12. (Color) Off-momentum beta beating for the lowbetamax collision optics with a relative momentum offset of 0.00027 (top); $z$-dependent beta beating due to the global crab cavity (bottom), which is much smaller than the off-momentum beta beating.
A frequency or phase jitter in the crab cavity can lead to a time-dependent beta beating. Also this $z$-dependent beta beating can be an additional source of synchrobetatron resonances. However, as the beta beating from the crab cavity is very small, it will not have a large impact on the beam dynamics.

\section{LUMINOSITY}

In this section we will introduce both the numerical simulation and the analytical treatment of the luminosity, for the crab crossing cases, with either two beams or one beam crabbed.

\section{A. GUINEA-PIG simulation}

GUINEA-PIG is a computer code for simulating the luminosity of colliders [15]. Here we apply this code to a storage ring, in order to simulate the single-bunch geometric luminosity at the LHC. Two bunches at the IP1 or IP5, each consisting of 10000 macroparticles, are generated with a Gaussian distribution in all six phase-space coordinates $(x, p x, y, p y, c t, \Delta p)$, for the LHC optics without crab cavity. The rms values for the Gaussian distributions are calculated from the design beam emittance, the design beam energy, and the design beta functions at the IP. We assume that the phase advance between crab cavity and the IP is exactly $\pi / 2$, and that $\alpha=0$ at both the IP and at the crab-cavity location, for the case with crab cavity. Then, applying formula (1), a horizontal kick from the crab cavity is added for each particle and a new bunch distribution is obtained [10].

Simulation results for the LHC luminosity under different conditions are compiled in Table III, for the local crab scheme (local scenario). For the nominal LHC optics without crab cavity, the simulated luminosity is in good agreement with the design value. A linearized crab cavity is used to check the validity of the transverse kick from the crab cavity.

For the global crab scheme with only beam 1 crabbed (single global scenario), a similar simulation is performed by using GUINEA-PIG. With only one horizontally crabbed beam, the simulated luminosity for nominal LHC collision optics is as follows: for IP5 (horizontal crossing), the luminosity is $1.04 \times 10^{34} \mathrm{~cm}^{-2} \mathrm{~s}^{-1}$; for IP1 (vertical

TABLE III. LHC luminosity $\left[10^{34} \mathrm{~cm}^{-2} \mathrm{~s}^{-1}\right]$ from GUINEAPIG simulation, with local crabbing scheme for both beams.

\begin{tabular}{lcc}
\hline \hline & LHC nominal collision & lowbetamax \\
\hline Design & 1.00 & \\
Head-on & 1.18 & 2.56 \\
Crossing & 1.00 & 1.50 \\
Linear crab crossing & 1.18 & 2.49 \\
400-MHz crab crossing & 1.17 & 2.43 \\
800-MHz crab crossing & 1.12 & 2.15 \\
\hline \hline
\end{tabular}


TABLE IV. Luminosity simulated by GUINEA-PIG for different global crabbing cases with only one global crab cavity [similar luminosity gain (IP5) and loss (IP1)].

\begin{tabular}{lcccc}
\hline \hline & \multicolumn{2}{c}{ Nominal LHC $\left[\mathrm{cm}^{-2} \mathrm{~s}^{-1}\right]$} & \multicolumn{2}{c}{ LHC upgrade $\left[\mathrm{cm}^{-2} \mathrm{~s}^{-1}\right.$ ] } \\
& IP5 & IP1 & IP5 & IP1 \\
\hline No CC & \multicolumn{2}{c}{$1.00 \times 10^{34}$} & \multicolumn{1}{c}{$1.50 \times 10^{34}$} \\
400-MHz CC (crabbing angle $\theta / 2)$ & $1.06 \times 10^{34}$ & $0.93 \times 10^{34}$ & $1.80 \times 10^{34}$ & $1.21 \times 10^{34}$ \\
800-MHz CC (crabbing angle $\theta / 2)$ & $1.04 \times 10^{34}$ & $0.96 \times 10^{34}$ & $1.71 \times 10^{34}$ & $1.32 \times 10^{34}$ \\
\hline \hline
\end{tabular}

crossing), the luminosity is $0.96 \times 10^{34} \mathrm{~cm}^{-2} \mathrm{~s}^{-1}$. As the luminosity for nominal crossing is $1 \times 10^{34} \mathrm{~cm}^{-2} \mathrm{~s}^{-1}$, we get around $4 \%$ gain at IP5 and around 4\% loss at IP1, with only one global crab cavity at IR4. For the lowbetamax optics, we repeat the simulation for different cases (frequency and crabbed angle). All results are summarized in Table IV. We observe that the crab cavity has a greater effect for smaller $\beta^{*}$ (larger crossing angle). The crossing angle has to be increased at lower $\beta^{*}$ without the crab cavity in place, in order to keep the same beam separations in units of sigma. This results in a larger loss of geometric overlap between the two beams which the crab cavity helps to restore.

\section{B. Analytical treatment and comparison}

The luminosity formulas for the crab crossing cases are derived (local scenario and single global scenario) analytically, to compare with the simulation results of GUINEAPIG. We start from the original formulas in [7], where the bunch distribution is assumed to be Gaussian in the longitudinal direction and also in the plane of the crossing angle, namely

$$
\begin{aligned}
& \rho_{x}(x)=\frac{1}{\sigma_{x} \sqrt{2 \pi}} \exp \left(-\frac{x^{2}}{2 \sigma_{x}^{2}}\right), \\
& \rho_{z}(z)=\frac{1}{\sigma_{z} \sqrt{2 \pi}} \exp \left(-\frac{z^{2}}{2 \sigma_{z}^{2}}\right) .
\end{aligned}
$$

The coordinate systems for the two beams are sketched in Fig. 13, where $x_{1}$ and $s_{1}$ are the horizontal and longitu-

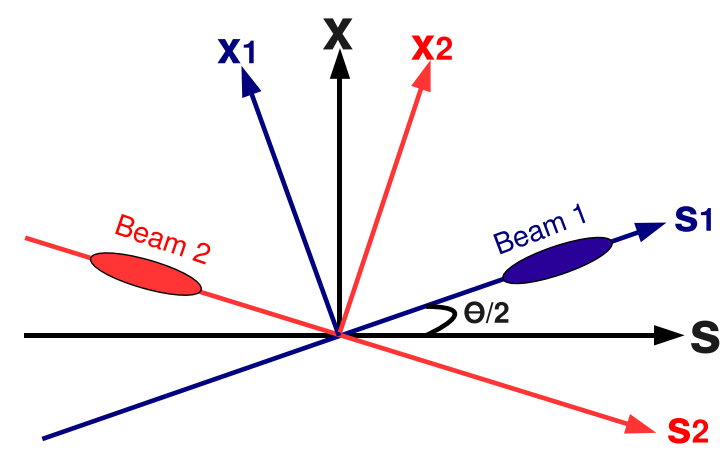

FIG. 13. (Color) Sketch of the coordinate system for the two colliding beams. dinal (along the beam line) coordinates of an arbitrary particle in beam 1; and $x_{2}$ and $s_{2}$ are the corresponding coordinates of an arbitrary particle in beam 2 . In comparison with the treatment in Ref. [7], here a more appropriate expression of the particle coordinates is used to correctly describe the kick from the crab cavity. We consider crab cavities operating at the rf wave number $k_{\mathrm{cr}}=2 \pi f_{\text {crab }} / c$. The voltage of the first local crab cavity is written as

$$
V_{1}=\frac{c^{2} \cdot p_{s} \cdot \tan \left(\frac{\theta}{2}\right)}{q \cdot \omega_{\mathrm{crab}} \cdot R_{12}}
$$

The change of the horizontal coordinate at the IP is then

$$
\Delta x_{1,2}= \pm R_{12} \cdot \frac{q V_{1}}{c \cdot p_{s}} \cdot \sin \left(\frac{2 \pi f_{\text {crab }}(s \mp c t)}{c}\right) .
$$

Taking the rf sinusoidal wave shape of the crab cavity into account, neglecting the $x$ dependent change of the particle energy, and also assuming $R_{22}=0$, the coordinates of the two colliding beams are written as

$$
\begin{gathered}
x_{1}=x \cos \frac{\theta}{2}-s \sin \frac{\theta}{2}+\frac{1}{k_{c r}} \sin \left[k_{\mathrm{cr}}(s-c t)\right] \sin \frac{\theta}{2}, \\
x_{2}=x \cos \frac{\theta}{2}+s \sin \frac{\theta}{2}-\frac{1}{k_{\mathrm{cr}}} \sin \left[k_{\mathrm{cr}}(s+c t)\right] \sin \frac{\theta}{2}, \\
s_{1}=s \cos \frac{\theta}{2}+x \sin \frac{\theta}{2}, \\
s_{2}=s \cos \frac{\theta}{2}-x \sin \frac{\theta}{2},
\end{gathered}
$$

where $\theta$ denotes the crossing angle, $c$ the velocity of light, and $k_{\mathrm{cr}}=2 \pi f_{\mathrm{crab}} / c$ the rf wave number of crab cavity. The third term $\Delta x_{1,2}$ on the right-hand side of formulas (12) and (13) describes the $x-z$ correlation resulting from the crab cavity.

The luminosity for head-on collision is given by

$$
L_{0}=\frac{N_{b}^{2} f_{\mathrm{rev}} n_{b}}{4 \pi \sigma_{x} \sigma_{y}}
$$

where $N_{b}$ denotes the number of particles inside a bunch, $f_{\text {rev }}$ the revolution frequency, $n_{b}$ the number of bunches per beam in total, $\sigma_{x}$ the horizontal rms bunch size at IP, and $\sigma_{y}$ the vertical rms bunch size at IP. 
The hourglass effect was neglected, and the corresponding luminosity with finite crossing angle can be expressed as

$$
\begin{aligned}
L= & \frac{c N_{b}^{2} f_{\text {rev }} n_{b}}{\sqrt{\pi} \sigma_{y}} \cos ^{2}(\theta / 2) \int_{-\infty}^{+\infty} \int_{-\infty}^{+\infty} \int_{-\infty}^{+\infty} \rho_{x}\left(x_{1}\right) \\
& \times \rho_{z}\left(s_{1}-c t\right) \rho_{x}\left(x_{2}\right) \rho_{z}\left(s_{2}+c t\right) d x d s d t
\end{aligned}
$$

Using formulas (8)-(15) and (17), for the two-beam crab crossing case, the luminosity reduction factor $R \equiv L / L_{0}$ in comparison with the head-on collision case can be expressed as

$$
\begin{aligned}
R_{2 \text {-beam }}= & \frac{\cos (\theta / 2) \cdot c}{\pi \cdot \sigma_{z}^{2}} \int_{-\infty}^{+\infty} \int_{-\infty}^{+\infty} \exp \left[-\frac{c^{2} t^{2}}{\sigma_{z}^{2}}-\frac{s^{2} \cos ^{2}(\theta / 2)}{\sigma_{z}^{2}}-\frac{\sin ^{2}(\theta / 2)}{4 k_{\mathrm{cr}}^{2} \sigma_{x}^{2}} \cdot\left\{2+4 k_{\mathrm{cr}}^{2} \cdot s^{2}-\cos \left[2 k_{\mathrm{cr}}(s-c \cdot t)\right]\right.\right. \\
& \left.\left.-\cos \left[2 k_{\mathrm{cr}}(s+c \cdot t)\right]-8 k_{\mathrm{cr}} \cdot s \cdot \cos \left(k_{c} \cdot c \cdot t\right) \sin \left(k_{c} \cdot s\right)-4 \cos ^{2}\left(k_{c} \cdot s\right) \cdot \sin ^{2}\left(k_{c} \cdot c \cdot t\right)\right\}\right] d t d s .
\end{aligned}
$$

We perform a numerical integration of (18) for $s$ in the range between minus infinity and plus infinity, and $c t$ in the range between minus $5 \sigma_{z}$ and plus $5 \sigma_{z}$, for the $400-\mathrm{MHz}$ and $800-\mathrm{MHz}$ crab-cavity cases, respectively. In Fig. 14 (left), the normalized luminosity from the analytical formula (18) is plotted with curves, and the simulation results from GUINEA-PIG are indicated with dots. Good agreement is found between these two. The smaller figure in Fig. 14 (left) shows a close-up view of the result near the nominal value $\beta^{*}=0.55 \mathrm{~m}$.

For the crabbing case with only one beam crabbed (single global scenario), the coordinate $x$ of beam 2 is written as

$$
x_{2}=x \cos \frac{\theta}{2}+s \sin \frac{\theta}{2} .
$$

The other coordinates stay the same as in formulas (12), (14), and (15).

Following a treatment similar to the above, the luminosity reduction factor $R \equiv L / L_{0}$ in comparison with the head-on collision case can be derived and expressed as

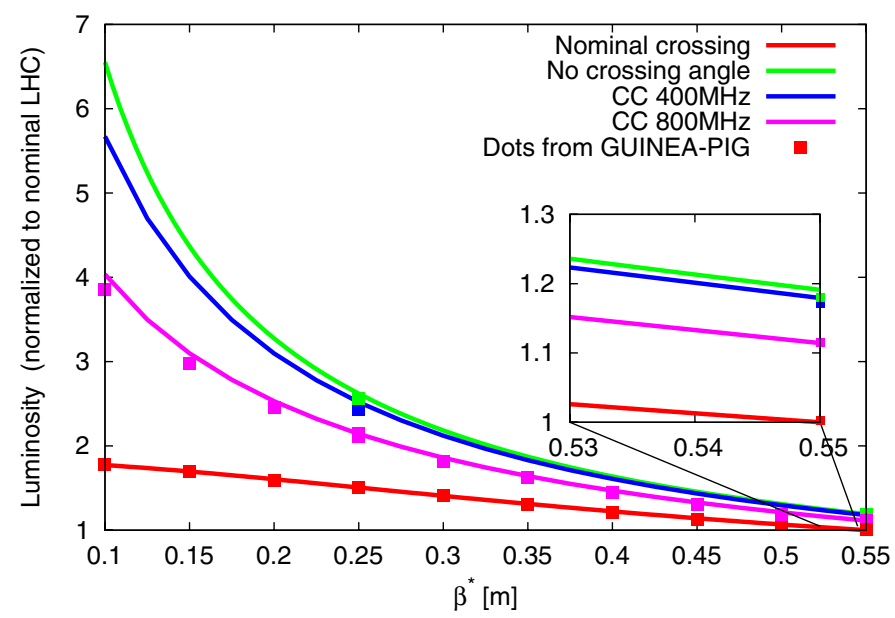

$$
\begin{aligned}
R_{1-\text { beam }}= & \frac{\cos (\theta / 2) \cdot c}{\pi \cdot \sigma_{z}^{2}} \int_{-\infty}^{+\infty} \int_{-\infty}^{+\infty} \\
& \times \exp \left[-\frac{c^{2} t^{2}}{\sigma_{z}^{2}}-\frac{s^{2} \cos ^{2}(\theta / 2)}{\sigma_{z}^{2}}\right. \\
& \left.-\frac{\sin ^{2}(\theta / 2)\left\{-2 k_{\mathrm{cr}} s+\sin \left[k_{\mathrm{cr}}(s-c \cdot t)\right]\right\}^{2}}{4 k_{\mathrm{cr}}^{2} \sigma_{x}^{2}}\right] d t d s .
\end{aligned}
$$

For the single global scenario, again we find good agreement between the analytical formulas and GUINEAPIG simulations, as is shown in Fig. 14 (right). Since we have neglected the hourglass effect in the analytical treatment, from Fig. 14 (left) and Fig. 14 (right) we observe that at smaller $\beta^{*}$ (for example $0.1 \mathrm{~m}$ ) the agreement between the analytical formulas and GUINEA-PIG simulations deteriorates.

For the single global scenario, the luminosity is also scanned versus different crabbing angle at IP5, both analytically and numerically with GUINEA-PIG. The results for

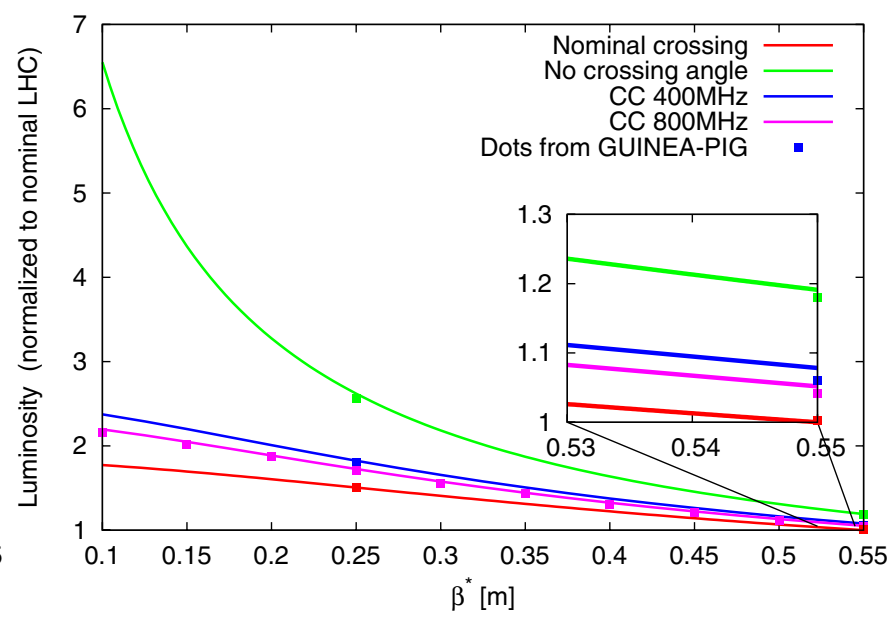

FIG. 14. (Color) Comparison of normalized luminosity between analytical formulas (curves) and GUINEA-PIG simulations (dots), with two horizontally crabbed beams (each by a crabbing angle of $\theta / 2$ ) at IP5 (left); and with only one horizontally crabbed beam (by a crabbing angle of $\theta / 2$ ) at IP5 (right). Simulations and analytical expressions agree. 

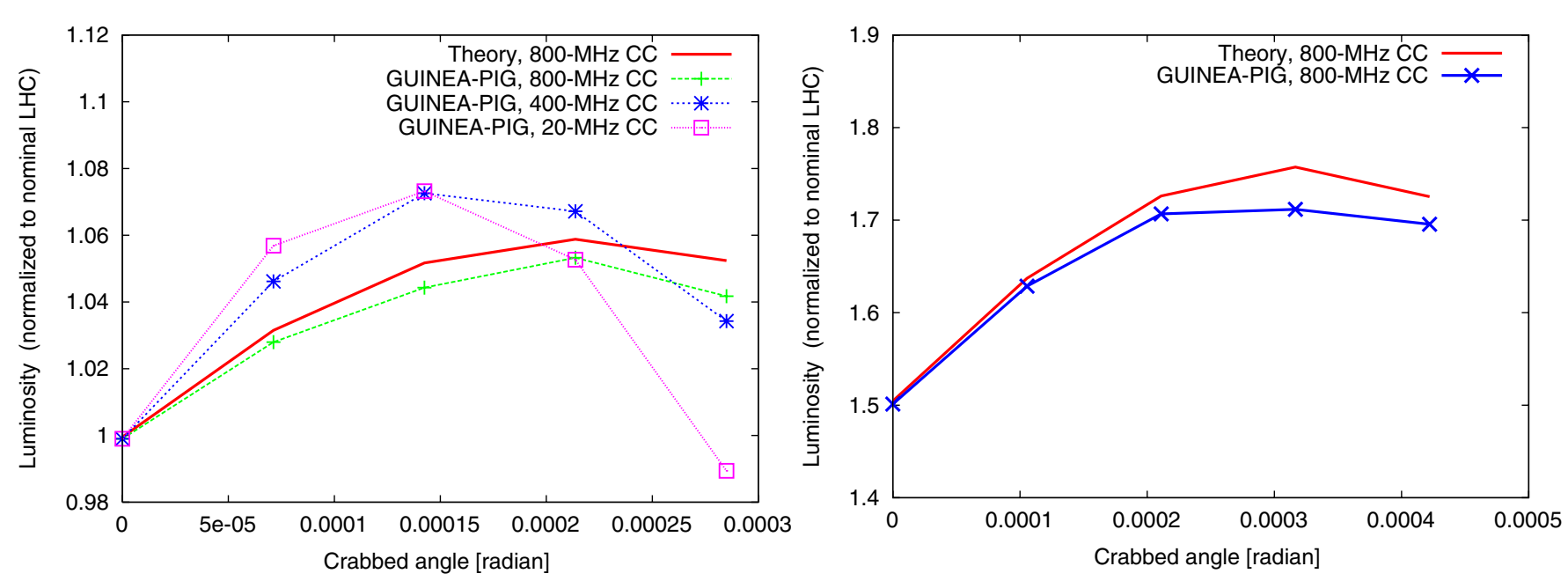

FIG. 15. (Color) Relative luminosity gain (normalized to the case without crab cavity) versus crabbing angle at IP5, for the nominal LHC collision optics (left) and for the lowbetamax collision optics (right) with a single global crab cavity. A luminosity gain of 7\% or $25 \%$ is achieved, respectively. For $20 \mathrm{MHz}$ the optimum crab angle equals half the crossing angle as theoretically expected while this is no longer the case for the higher crab frequencies due to the rf curvature.

the nominal LHC collision optics in Fig. 15 (left) show that the luminosity (normalized to $10^{34} \mathrm{~cm}^{-2} \mathrm{~s}^{-1}$ ) assumes a maximum as a function of the crabbing angle, the location of which depends on the rf frequency of the crab cavity. For example for the $800-\mathrm{MHz}$ crab-cavity case, the peak is around three-quarters of the full crossing angle, and the analytical result agrees with the GUINEA-PIG simulation. At the same time for the $20-\mathrm{MHz}$ crab-cavity case, the peak is at half of the full crossing angle, as one would expect for the ideal linear case. The shift of the optimum crabbing angle towards larger values for higher crab rf frequencies

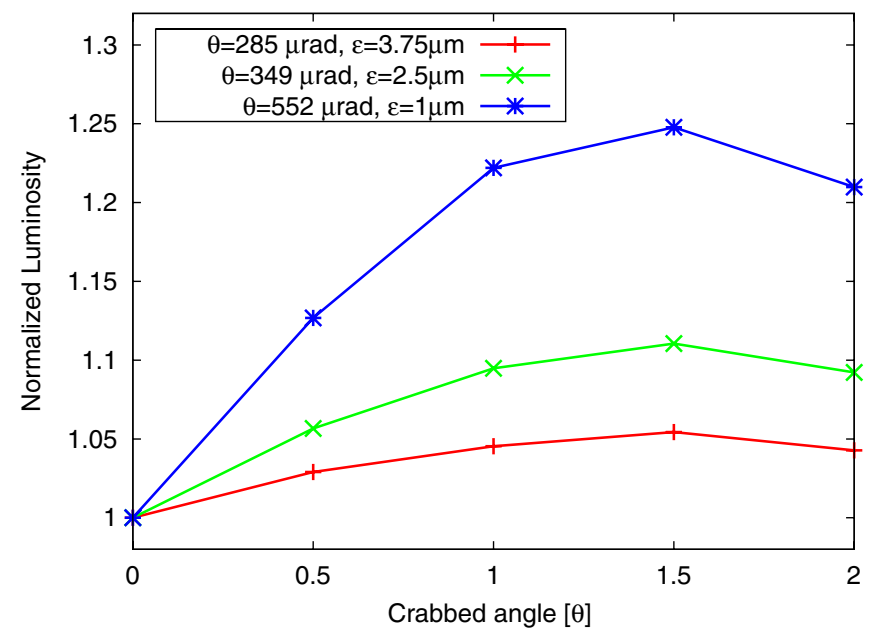

FIG. 16. (Color) Relative luminosity gain (normalized with the corresponding crossing case and the emittance set to 3.75, 2.5, and $1 \mu \mathrm{mrad}$ for the three cases, respectively) for three cases with different beam emittance and bunch intensity (nominal LHC with $\beta^{*}=0.55 \mathrm{~m}, \theta=285-552 \mu \mathrm{rad}$ ), from GUINEAPIG simulations with a single global crab cavity. The luminosity gain is $10 \%-25 \%$ for lower emittance. can be explained by the fact that due to the nonlinear curvature of the rf wave most of the bunch experiences a lower deflection than for the ideal linear crab cavity. Then, to optimize the overlap of the two bunches, a larger crab voltage, or equivalent crab angle, is required, so that over the length of the bunch the "average" value of the nonlinear crab deflection is close to the optimum value of $1 / 2$ crossing angle. Optimizing the overlap of the two colliding beams, or equivalently maximizing $R$ in formula (20), determines the location of the maximum.

If the LHC luminosity can be measured with a resolution of $1 \%$, as expected [16], it will be possible to detect the effect of this global crab cavity, during the very first test of a single crab cavity in the LHC. A similar scan is performed for the lowbetamax collision optics with $\beta^{*}=$ $0.25 \mathrm{~m}$ at IP1 and IP5 (and with one $800-\mathrm{MHz}$ global crab cavity), and the corresponding result is shown in Fig. 15 (right).

To obtain a larger luminosity gain from crab crossing in the test phase (single global scenario), one can use a beam with much smaller emittance and lower bunch intensity. As illustration we consider three different beam emittances for the nominal LHC with $\beta^{*}=0.55 \mathrm{~m}$, as $\epsilon=3.75 \mu \mathrm{m} \mathrm{rad}$, $\epsilon=2.5 \mu \mathrm{m} \mathrm{rad}$ and $\epsilon=1 \mu \mathrm{m} \mathrm{rad}$, respectively (which is already available from LHC injectors, for the LHC TOTEM experiment). The luminosity gain versus crabbing angle at IP5 is shown in Fig. 16, where we observe that for the case with $\epsilon=1 \mu \mathrm{m}$ rad the luminosity gain can be as large as $25 \%$.

\section{EMITTANCE GROWTH}

The transverse kick from the global crab cavity excites betatron oscillations of the beam if the voltage ramp of the crab cavity is not adiabatic, potentially increasing the 
intrinsic transverse emittance (in which case the increase of the projected emittance remaining even after the crab cavity has been ramped down). It is important to study how slow the ramping up speed of the crab cavity should be, in order to have a negligible influence on the intrinsic beam emittance. Morita has performed some studies by using the SAD code and a 400-MHz global crab cavity [17]. Here a more detailed study is performed with the code MADX [18], by modifying the thintrack module [10] for the LHC nominal collision optics.

Ten-thousand particles in a 6D Gaussian distribution are generated, and the beam parameters such as the unnormalized emittance $0.5 \mathrm{~nm}, \beta^{*}=0.55 \mathrm{~m}$, beam energy, bunch length $\sigma_{z}=0.075 \mathrm{~m}$, and rms energy offset $\sigma_{p}=1.1 \times$ $10^{-4}$ are taken into account. The tracking is done turn by turn for thousands of turns. On each turn the emittance and bunch size are calculated by using the statistical formulas.

The $800-\mathrm{MHz}$ global crab cavity, which is described in the above section, is ramped up from turn 0 , over $1,5,10$, 100, and 1000 turns, respectively. Then between 2000 and 2100 turns, the crab-cavity voltage is ramped down within 100 turns. To check the effect of the crab cavity ramping, one particle with zero transverse and longitudinal offset, and $2.5 \sigma_{p}$ energy offset was tracked for 500 turns before ramping, 5000 turns while ramping, and 500 turns after ramping. One BPM is placed at IP5 to record the position of this particle for each turn. The simulated longitudinalhorizontal correlation during synchrotron oscillation periods while ramping the crab-cavity voltage is shown in Fig. 17, where it can be seen that the time-dependent cavity ramping is correctly implemented in the code.

The horizontal emittance for different cases is shown in Fig. 18, where it can be seen that for a ramping up time which is longer than 10 turns, the emittance can be recovered after the crab-cavity voltage is ramped down. When the crab cavity is on, the projected emittance growth is

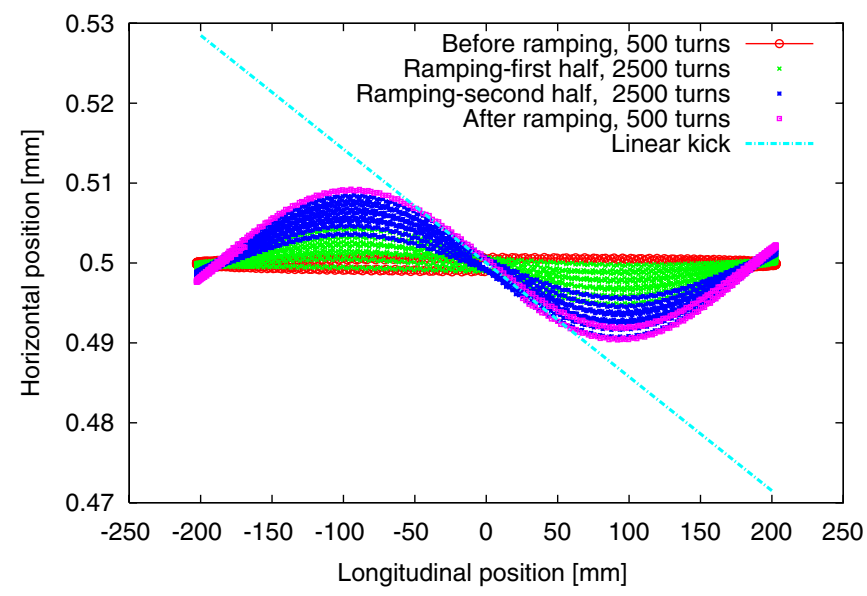

FIG. 17. (Color) Check of crab cavity ramping by tracking reveals a ramp-up time of 5000 turns is sufficiently adiabatic with respect to the synchrotron motion.

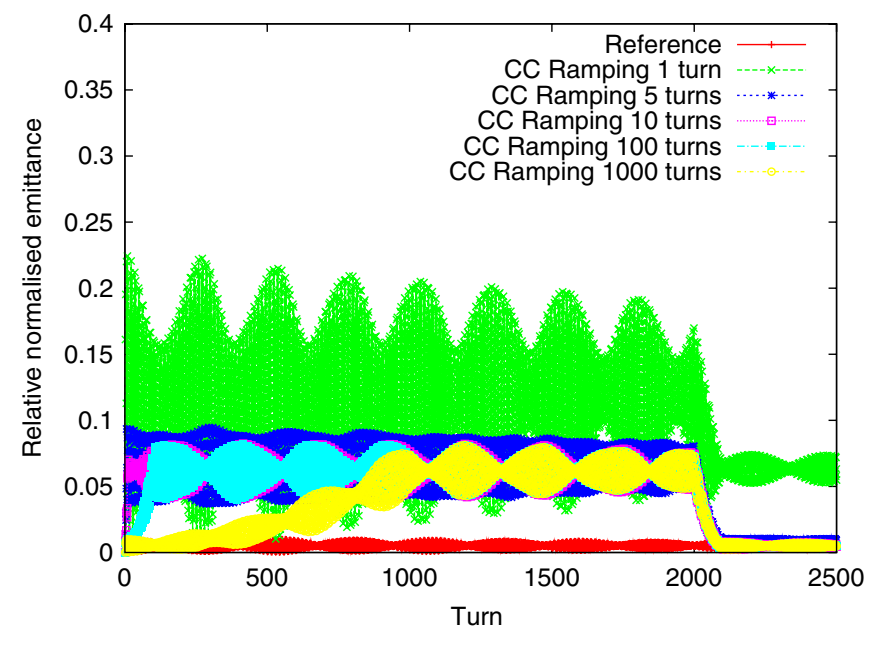

FIG. 18. (Color) Relative horizontal emittance growth for different ramping speed of one $800-\mathrm{MHz}$ global crab cavity. A ramping period longer than 10 turns is required to avoid the emittance growth.

between $6 \%$ and $13 \%$. For the 1 turn ramping up case, the horizontal emittance remains increased by $6 \%$ after the crab cavity has been ramped down. The larger projected emittance reflects the desired effect of the crab cavity to introduce $x-z$ correlation.

The $400-\mathrm{MHz}$ global crab-cavity case has also been checked, and the results confirm the SAD results of Morita [17]. As the crab cavity primarily kicks the beam in the horizontal direction, the vertical and the longitudinal emittance should not be affected. This is confirmed by our simulation.

In reality, there will be many other disturbing nonlinear terms, in addition to the chromatic sextupoles considered above. To explore their effect, we also include the effects of the nominal beam-beam kick (at all four IPs), the nonlinear magnetic fields error (same as those for the dynamicaperture studies), and all the Landau octupoles, to observe the impact of nonlinear fields and the associated tune spread on the emittance growth. No obvious emittance growth is found when tracking with these nonlinear sources alone, and no obvious difference is found for the crab-cavity ramping cases with and without these nonlinear elements. The emittance fluctuation from the Landau octupoles alone, with a strength of 16 and $100 \mathrm{~m}^{-4}$, respectively (corresponding to tune shifts of $2 \times$ $10^{-5}$ and $1 \times 10^{-4}$, for $1 \sigma$ offset), is due to numerical noise.

In principle, there also is a possibility to use a crab cavity with another frequency for the LHC upgrade, such as $400 \mathrm{MHz}$. A comparison of the emittance growth, from the crab cavity ramping with crab cavities of different frequency (20, 400, and $800 \mathrm{MHz})$, and with 1 turn ramping speed is shown in Fig. 19 (left). In Fig. 19 (right) a similar comparison is repeated, with all the Landau octupoles strength set to be $16 \mathrm{~m}^{-4}$. It is clearly seen that the 

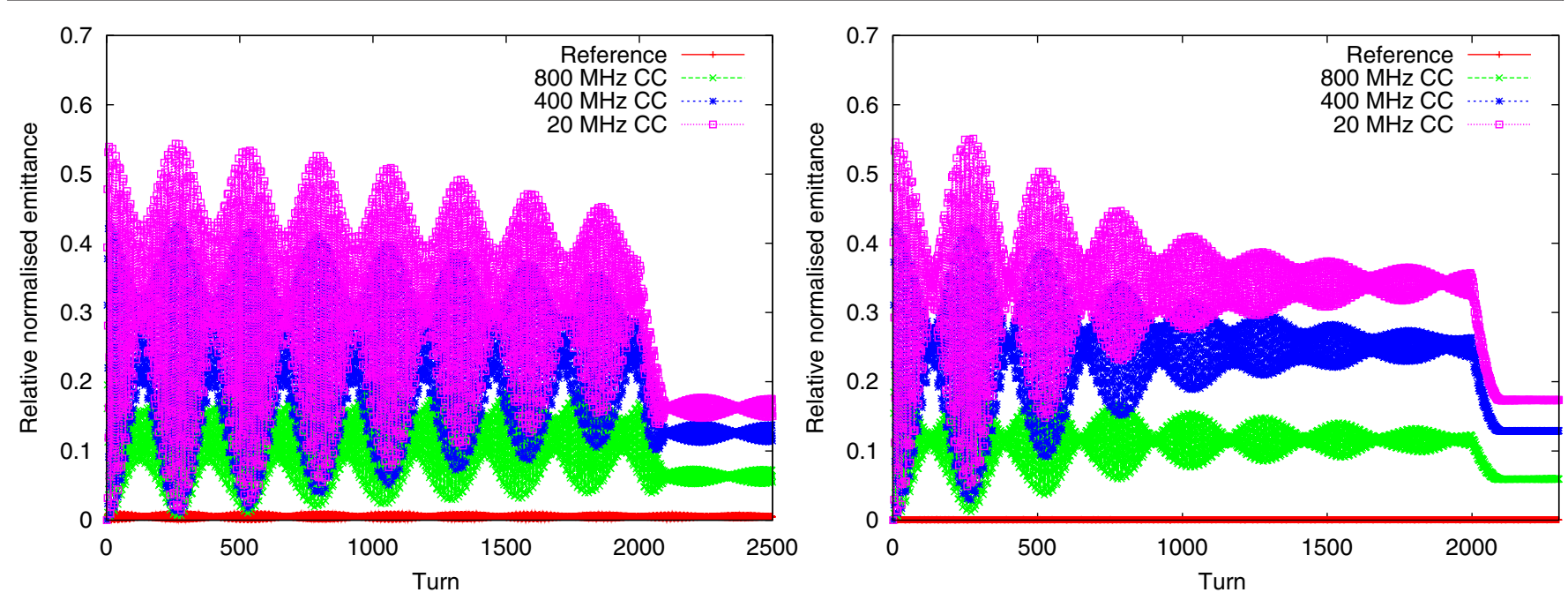

FIG. 19. (Color) Relative horizontal emittance growth due to the crab cavity ramping with different frequency crab cavities (20, 400, and $800 \mathrm{MHz}$ ), without (left) and with (right) Landau octupoles, with 1 turn ramping speed. The lower the frequency is, the larger the emittance growth will be.

lower the frequency is, the larger the emittance growth will be. With Landau octupoles, the transverse oscillations are damped faster, as it is expected to be. However, the residual emittance growth visible after the crab cavity being ramped down is similar with and without octupoles.

The crab cavities with different frequency (linear, 400 and $800 \mathrm{MHz}$ ) all impart a similar linear kick on the beam, within the $\pm 1 \sigma_{z}$ range. So if a longitudinal cut on the initial distribution is performed at $1 \sigma$, the emittance growth from the crab cavity ramping with different frequency crab cavities should be similar. That is confirmed by tracking and shown in Fig. 20. To mitigate the emittance growth, the ramping period of the crab cavities will have to be larger than 10 turns.

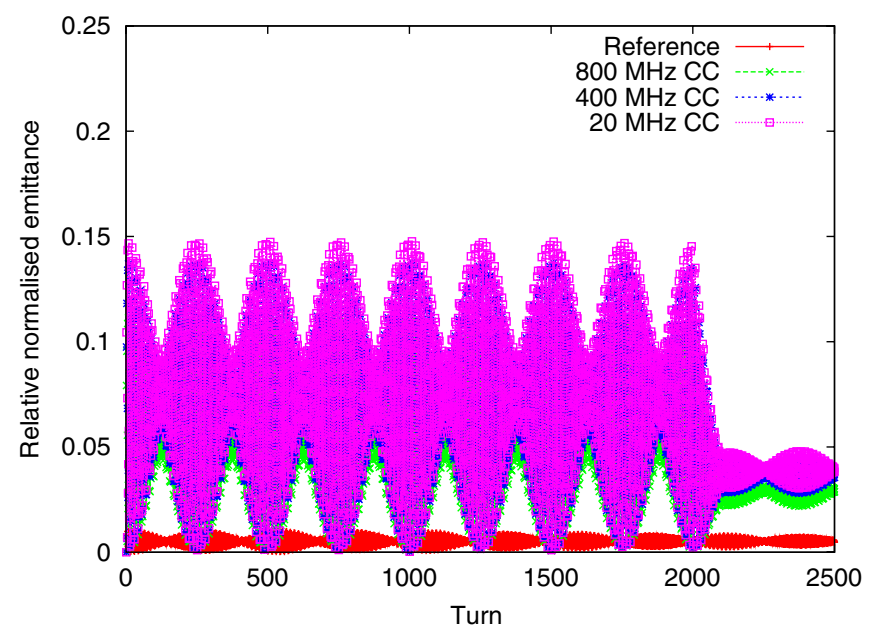

FIG. 20. (Color) Relative horizontal emittance growth due to the crab cavity ramping with different frequency crab cavities $(20$, 400 , and $800 \mathrm{MHz}$ ), longitudinal cut at $1 \sigma$, with 1 turn ramping speed. The transverse emittance growth is due to particles in the longitudinal tails.

\section{CRAB DISPERSION}

The crab cavity's effect is like an orbit corrector and the difference to a true orbit corrector is that the kick is $z$ dependent. So following the approach of the closed-orbit distortion, we can get the deviation of the crab closed orbit with a specified longitudinal position along the bunch as [19]

$$
\begin{aligned}
x_{D_{c c}}(z, s)= & \sqrt{\frac{\beta(s)}{\beta^{*}}} \cdot \frac{c \cdot \tan \left(\frac{\theta}{2}\right)}{\omega} \cdot \sin \left(\frac{\omega z}{c}\right) \\
& \cdot \frac{\cos \left(\Delta \varphi_{1}-\pi Q\right)}{\cos \left(\Delta \varphi_{0}-\pi Q\right)},
\end{aligned}
$$

where $\theta / 2$ denotes the half crossing angle (crabbing angle for each beam), $\omega$ the angular rf frequency of the crab cavity, $z$ the longitudinal coordinate of the particle with respect to the bunch center, $\Delta \varphi_{1}$ the phase advance between the crab-cavity location and the location $s$ in the ring, and $\Delta \varphi_{0}$ the phase advance between the crab-cavity location and the IP.

A particle which only has $z=1 \sigma_{z}$ as initial offset will later be at $\delta_{p}=1 \sigma_{p}$ and $z=0$, due to synchrotron oscillation. Also to arrive at a definition with the same units as the off-momentum dispersion, for the case $c / \omega \gg \sigma_{z}$ (linear crab kick within $\pm 1 \sigma_{z}$ ), we define the "crab dispersion" as the orbit deviation at $z=1 \sigma_{z}$ normalized to $1 \sigma_{p}[19]:$

$$
D_{c c}(s)=\frac{x_{D_{c c}}\left(1 \sigma_{z}, s\right)}{1 \sigma_{p}} .
$$

Applying formulas (21) and (22) to the global crabbing scheme (single global scenario), we get the crab dispersion shown in Fig. 21, for the nominal LHC collision optics. At the specified IP (IP5), we get the required crab dispersion 


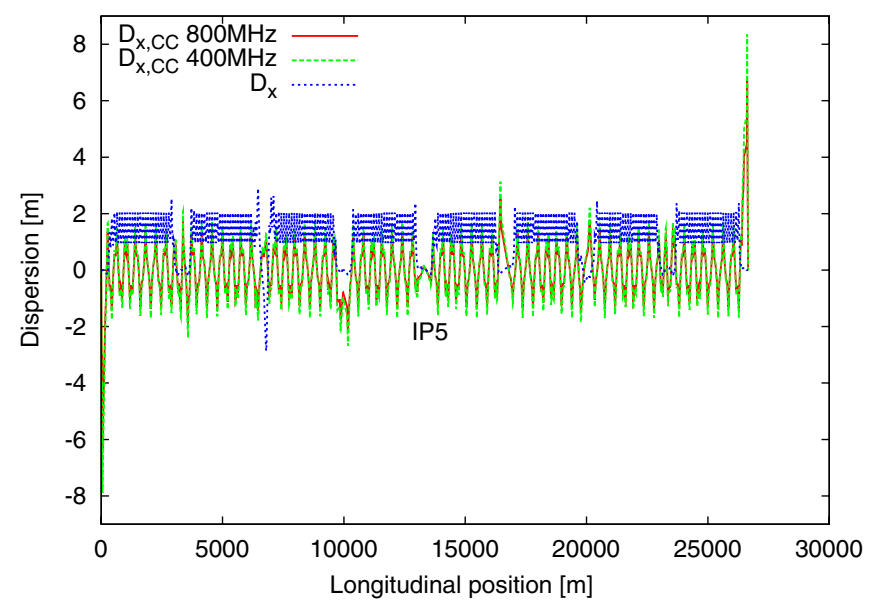

FIG. 21. (Color) Crab dispersion (IP5 is at $13329 \mathrm{~m}$ ) due to one 800-MHz global crab cavity (red) and one 400-MHz global crab cavity (green); normal dispersion (blue).

(value of $8.3 \times 10^{-2} \mathrm{~m}$ ) to achieve the quasi-head-on collision.

\section{COLLIMATION}

The LHC collimation system is designed to protect the cold magnets and to absorb the beam halo outside of a specified transverse beam size. There are two main regions where LHC collimators are concentrated: IR3 (momentum cleaning) and IR7 (betatron cleaning). Several kinds of collimators exist in the LHC. Among these the two main groups are the primary collimators and the secondary collimators. The difference between the jaw opening of the primary and secondary collimators is about one beam sigma. Given the condition that the secondary collimators should never be hit by the primary beam halo, the closed orbit shall be strictly controlled in order to set the right jaw opening of collimators. As the global crab cavity changes the particles' $z$-dependent "closed orbit," it is essential to consider how the crab cavity will affect the collimation system.

The closed orbit at different $z$ offsets is shown in Fig. 22 (which starts at IP3), for the nominal LHC optics with one $800-\mathrm{MHz}$ global crab cavity located at IR4. It can be seen that at some collimator locations, the orbit difference between particles launched at $1 \sigma_{z}$ and $2 \sigma_{z}$ (or $2 \sigma_{z}$ and $3 \sigma_{z}, \ldots$ ) can be as large as one sigma or more, and that the difference to the nominal orbit stays below $1 \sigma$ for all the collimators. This is neither insignificant nor large enough to rule out the use of global crab cavities.

The cold elements of LHC could be damaged by the halo particles that escape the collimators (primary, secondary, ...). Therefore it is important to define and study the cleaning efficiency of the collimation system. The "global cleaning inefficiency" is defined as the leakage rate for a specified aperture $A_{c}$ [20]. However, even if the global cleaning inefficiency $\eta_{c}$ (for $A_{c}=10 \sigma$ ) is under $10^{-3}$

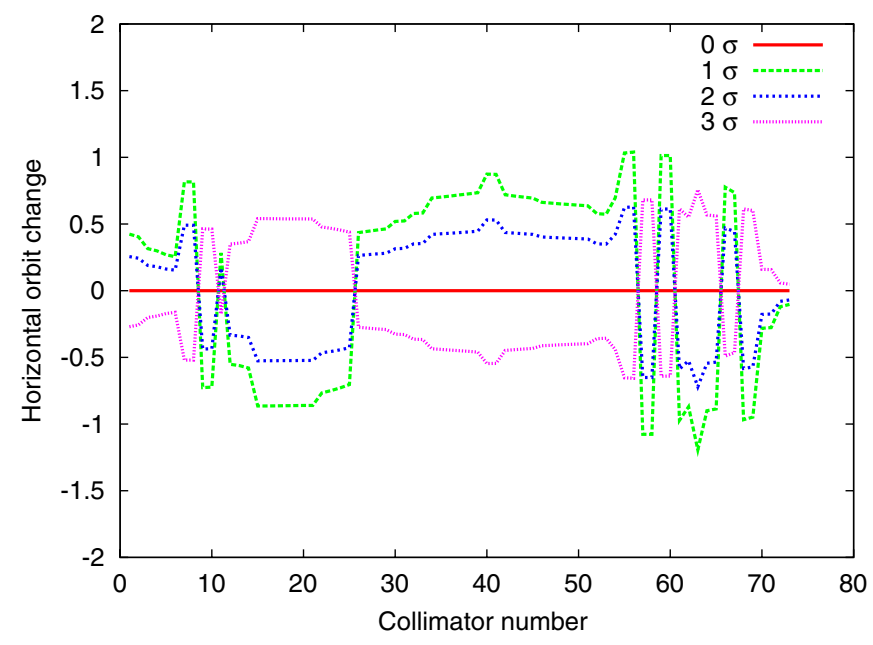

FIG. 22. (Color) $z$-dependent closed-orbit change induced by the global crab cavity in IR4. The maximum orbit change is about $1 \sigma$.

which is the limit for the LHC at top energy, the cold magnets could still be quenched by the local concentration of the particle losses. Based on this, it is much more important to study the distribution of the halo particles along the ring, and the local cleaning inefficiency is defined as [20]

$$
\tilde{\eta}_{c}=\frac{\eta_{c}}{L_{\mathrm{dil}}},
$$

where $L_{\mathrm{dil}}$ is the dilution length of the losses with a resolution up to $10 \mathrm{~cm}$.

In this paper, the collimation simulation results presented are done for the on-momentum beam halo which has no energy offset and longitudinal offset. As the global crab cavity applies a $z$-dependent kick, we study several different cases with beam halo particles located in different longitudinal slices of the bunch (with a fixed longitudinal position) [19], considering the horizontal and vertical beam halo, respectively. The collimators are set with respect to the center of the vacuum chamber (not the $z$-dependent closed orbit). Here we mainly introduce the result for the worst case $z=1 \sigma_{z}$ and with horizontal beam halo.

To compare with the nominal local cleaning inefficiency results of the collimation team, we use all the same tracking setups as developed for collimation studies for the nominal LHC [21]. To have a $1 \mu \mathrm{m}$ impact parameter [21], the beam halo is generated at $5.509 \sigma$ with $0.0015 \sigma$ as smear [19]. We analyze the location of particle losses along the accelerator, and this is done with comparison between the aperture and the particles' trajectories. For the case with horizontal beam halo, the particles impact with the primary collimator "TCP.C6L7.B1" at the first turn. The impact parameter, which is defined to be the impact depth of halo particles from the edge of the collimator jaws when they interact with the jaw material (as shown in 


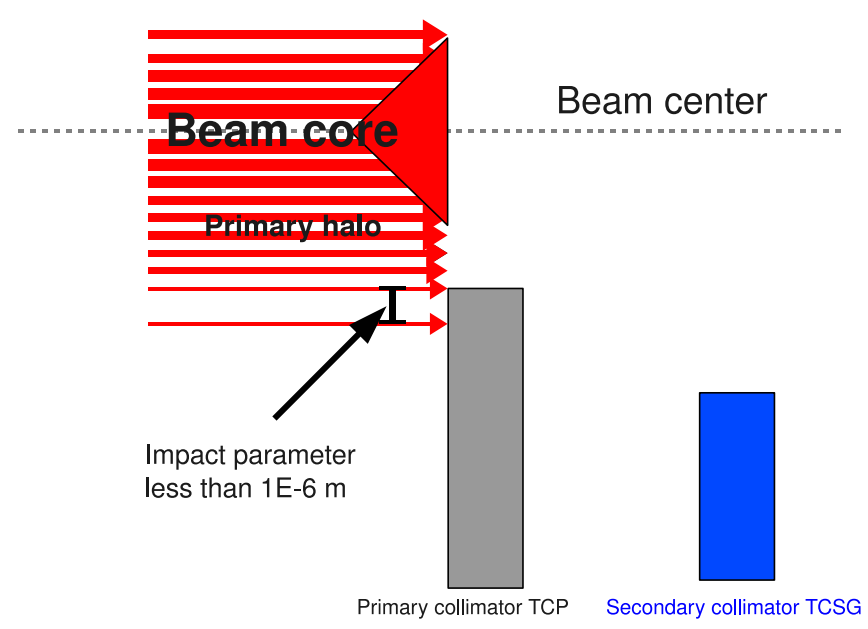

FIG. 23. (Color) Schematic of the impact parameter on the LHC primary collimator.

Fig. 23), is averaged over all the particles which impact with the collimator for the first time at the specified turn, as shown in Fig. 24. We observe that the impact parameter is modulated by the betatron oscillations and reaches its maximum around turn 20.

The local cleaning inefficiency (with a longitudinal resolution down to $10 \mathrm{~cm}$ ) is shown in Fig. 25 for particles hitting the IR7 primary collimators (beam halo generated at $\left.z=1 \sigma_{z}\right)$. Overall, most of the halo particles are absorbed by the collimators and a loss on the cold magnets occurs mainly in the dispersion suppressor downstream of IP7. Furthermore, if we look at the local loss map of the case with global crab cavity, we find that it is similar to the case without crab cavity, and for both cases the cold loss rates remain mostly still below the quench limit.

We also consider the crab cavity's impact on the available phase space for the circulating beam at each collimator ( $z$-dependent crab dispersion). With crab dispersion

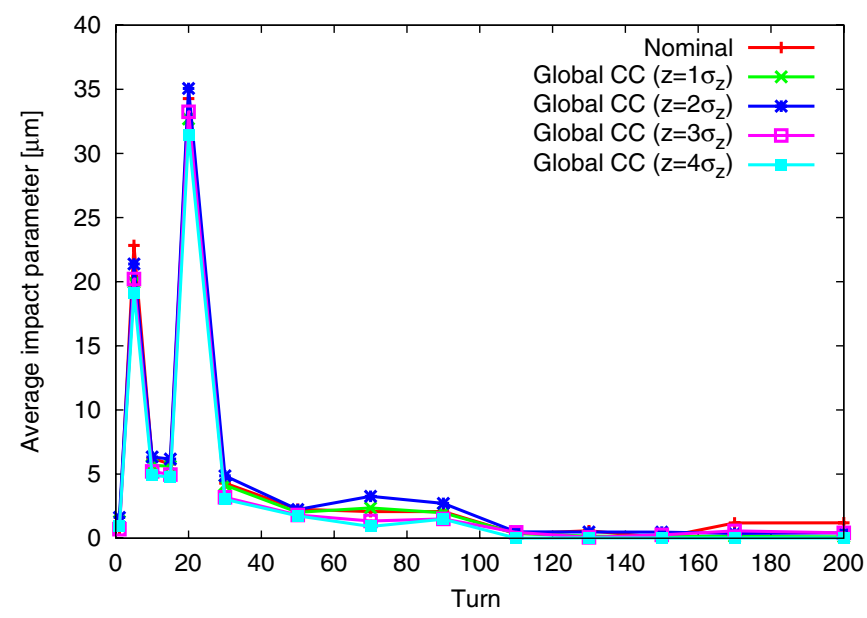

FIG. 24. (Color) Average first-time impact parameter at specified turn for different cases. All cases have a $1 \mu \mathrm{m}$ impact parameter for the first turn.

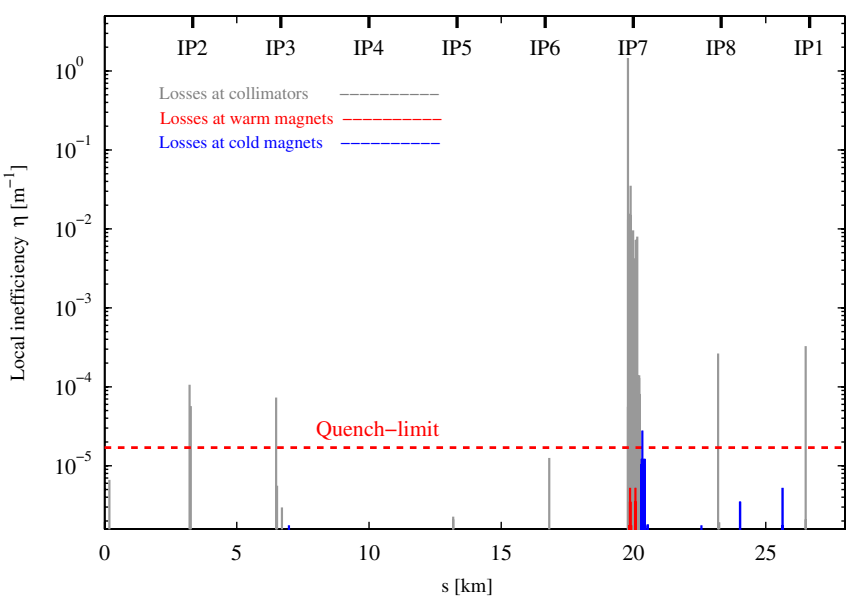

FIG. 25. (Color) Local loss map for nominal LHC (top energy, $\beta_{\mathrm{IP} 1,5}^{*}=0.55 \mathrm{~m}$ ), with one global crab cavity and horizontal beam halo (beam halo generated at $z=1 \sigma_{z}$ ). A beam lifetime of $0.2 \mathrm{~h}$ is assumed to define the quench limit (red dashed line). This loss map is almost indistinguishable from the case without crab cavity.

included, the effective betatron amplitude cut at the $i_{\text {th }}$ collimator can be written as $[19,21]$

$$
\begin{aligned}
n_{\beta, \mathrm{cut}}\left(i_{\mathrm{coll}}, \delta, z\right)= & \frac{1}{\sqrt{\epsilon_{r} \beta_{r}\left(i_{\mathrm{coll}}, \delta\right)}}\left[ \pm r_{\mathrm{cut}}\left(i_{\mathrm{coll}}\right)\right. \\
& \left.-D_{r}\left(i_{\mathrm{coll}}, \delta\right) \cdot \delta-x_{D_{c c}}(z, s)\right],
\end{aligned}
$$

where $r_{\text {cut }}\left(i_{\text {coll }}\right)$ denotes the half gap of the collimator jaw at the $i_{\text {th }}$ collimator, $n_{\beta, \text { cut }}\left(i_{\text {coll }}, \delta\right)$ the effective betatron amplitude cut at the $i_{\text {th }}$ collimator, $\delta=\Delta p / p$ the energy offset, $\epsilon_{r}$ the beam transverse emittance, $\beta_{r}\left(i_{\text {coll }}, \delta\right)$ the beta function at the $i_{\text {th }}$ collimator, $D_{r}\left(i_{\text {coll }}, \delta\right)$ the dispersion at the $i_{\text {th }}$ collimator, $x_{D_{c c}}(z, s)$ the deviation of the closed orbit due to the $z$-dependent crab dispersion, $z$ the longitudinal coordinate of the particle with respect to the bunch center, and $s$ the longitudinal location in the ring.

If we only include the crab dispersion (as discussed in the above section), and do not include the off-momentum beat, we can get a phase-space cut for the circulating beam, illustrating the hierarchy of primary (TCP), secondary (TCSG), tertiary (TCTH), beam dump (TCDQ) horizontal collimators, and shower absorbers (TCLA) (which is preserved from the case without crab cavity), as shown in Fig. 26 [19]. The half gap of the primary (secondary) betatron collimator (in IR7) is $6 \sigma(7 \sigma)$ and the half gap of the primary (secondary) momentum collimator (in IR3) is $15 \sigma(18 \sigma)$. The opening of the different group of collimators could be inferred from Fig. 26.

Furthermore, a general longitudinal amplitude can be defined to combine the off-momentum dispersion and crab dispersion together [19], as $A_{z}=\sqrt{\delta_{p}^{2}+\tilde{\delta}_{z}^{2}}$, where $A_{z}$ denotes the general amplitude, $\delta_{p}$ energy offset, $\tilde{\delta}_{z}$ from the crab cavity. For simplicity, the most pessimistic case 


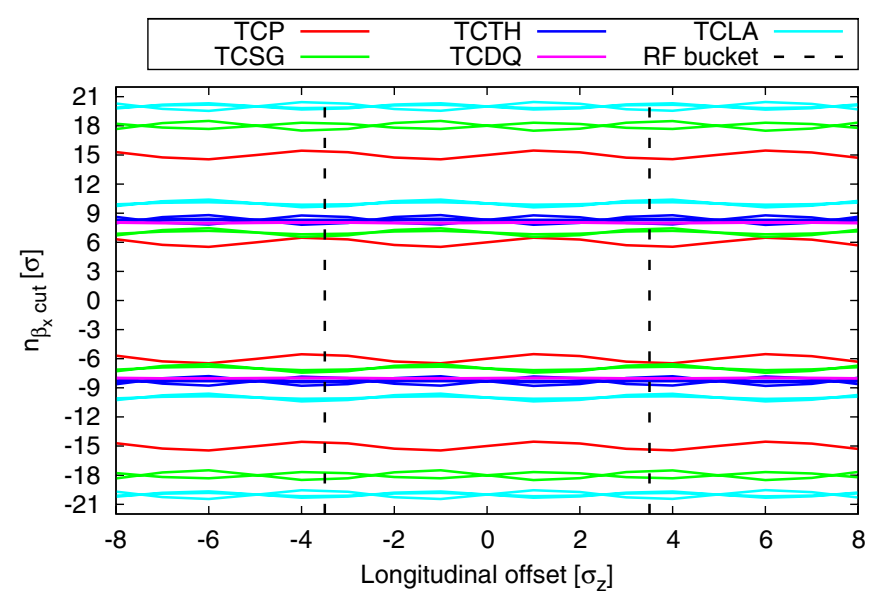

FIG. 26. (Color) Phase-space cut by various collimators in the presence of crab dispersion but zero momentum dispersion, with the hierarchy of primary (TCP), secondary (TCSG), tertiary (TCTH), beam dump (TCDQ) horizontal collimators, and shower absorbers (TCLA). $D_{r}\left(i_{\text {coll }}, \delta\right)=0$ in formula (24).

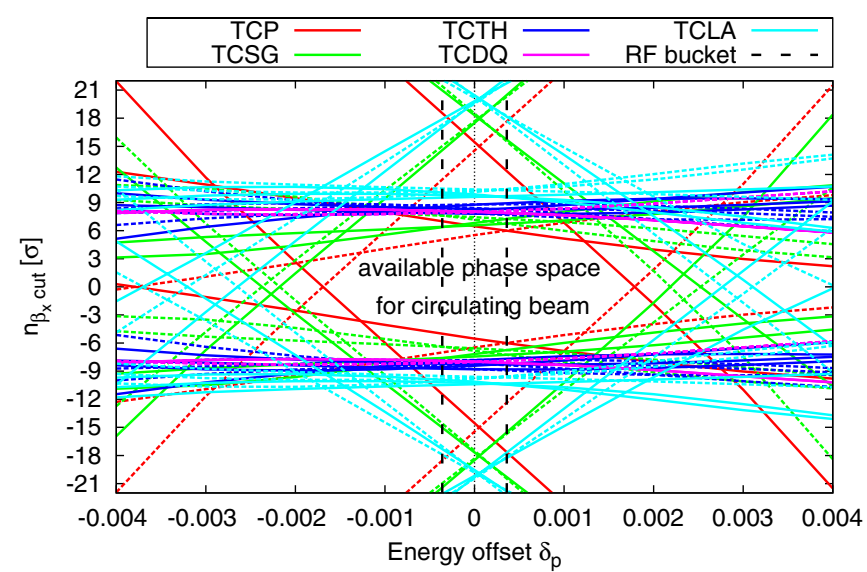

FIG. 27. (Color) CC disturbed phase space for the circulating beam by applying formula $(24)\left(z=1 \sigma_{z}\right)$, with the hierarchy of primary (TCP), secondary (TCSG), tertiary (TCTH), beam dump (TCDQ) horizontal collimators, and shower absorbers (TCLA) maintained. The white region labeled in the middle is the phase space available to the circulating beam.

from Fig. 26 (crab dispersion at $1 \sigma_{z}$ ) is used to construct the CC disturbed phase-space cut, as shown in Fig. 27. In this case the available phase space for the circulating beam is decreased by half a $\sigma$ at the most, compared with the nominal case without crab cavity [21].

\section{CRAB CROSSING TUNE SHIFT AND SYNCHROBETATRON RESONANCES}

To calculate the beam-beam tune shift of the crab collision case, we use a weak-strong model, a modified version of Hiratas's Lorentz boost where the crabbed strong beam in the c.m. frame is untilted [22], and follow the crossing angle treatment implemented in the SIXTRACK code by
Leunissen et al. [23]. The calculation of the beam-beam force is done by approximating the strong bunch by a number of longitudinal slices. The synchrobeam mapping originally formulated for collisions with a crossing angle and no crabbing [24] is then applied at the IP. For the SIXTRACK simulations, two local linear crab cavities modeled by formulas (1) and (3) with a low frequency are placed at both sides of IP5 to recover the head-on collision at IP5. The tracking is done with beam 1 (weak beam) represented by test particles, and beam 2 treated as the strong beam represented by the beam-beam element. The crabbing of the strong beam is modeled in the Lorentzboosted frame by modifying the beam-beam force, and the crabbing of the weak beam is done by a real crab cavity in the LHC optics. For the full crab collision case (both beams crabbed), the simulated linear tune shift is found to be the same as the corresponding head-on tune shift [25]. The tune shift for the crab collision case with only one beambeam element placed at IP5 and horizontal crossing is shown in Fig. 28.

In addition to first-order synchrobetatron sidebands due to nonzero chromaticity, second-order synchrobetatron sidebands are excited by different transverse beam-beam forces at different longitudinal positions (betatron tune modulated at twice the synchrotron frequency) in the colliding bunches, an effect which is introduced by the transverse crossing angle at the IP [26]. It was suggested that crab cavities could eliminate this kind of resonance for circular colliders [5]. We have studied the strength of the synchrobetatron resonances in simulations. Three cases are compared, namely, the beam-beam effect with a crossing angle (crossing collision), the crossing collision case plus
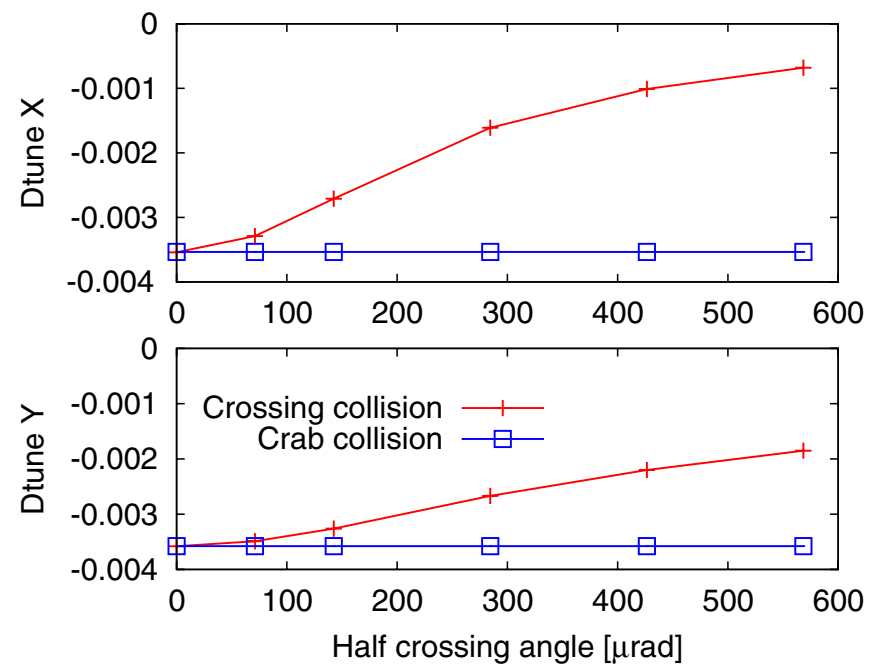

FIG. 28. (Color) Horizontal tune shift (top) and vertical tune shift (bottom): crossing collision with one beam-beam element at IP5(H) and horizontal crossing (red); full crab collision with the same beam-beam element at IP5 $(\mathrm{H})$ and both beam crabbed (blue). The crab crossing tune shift is found to be the same as the one for the head-on collision case. 

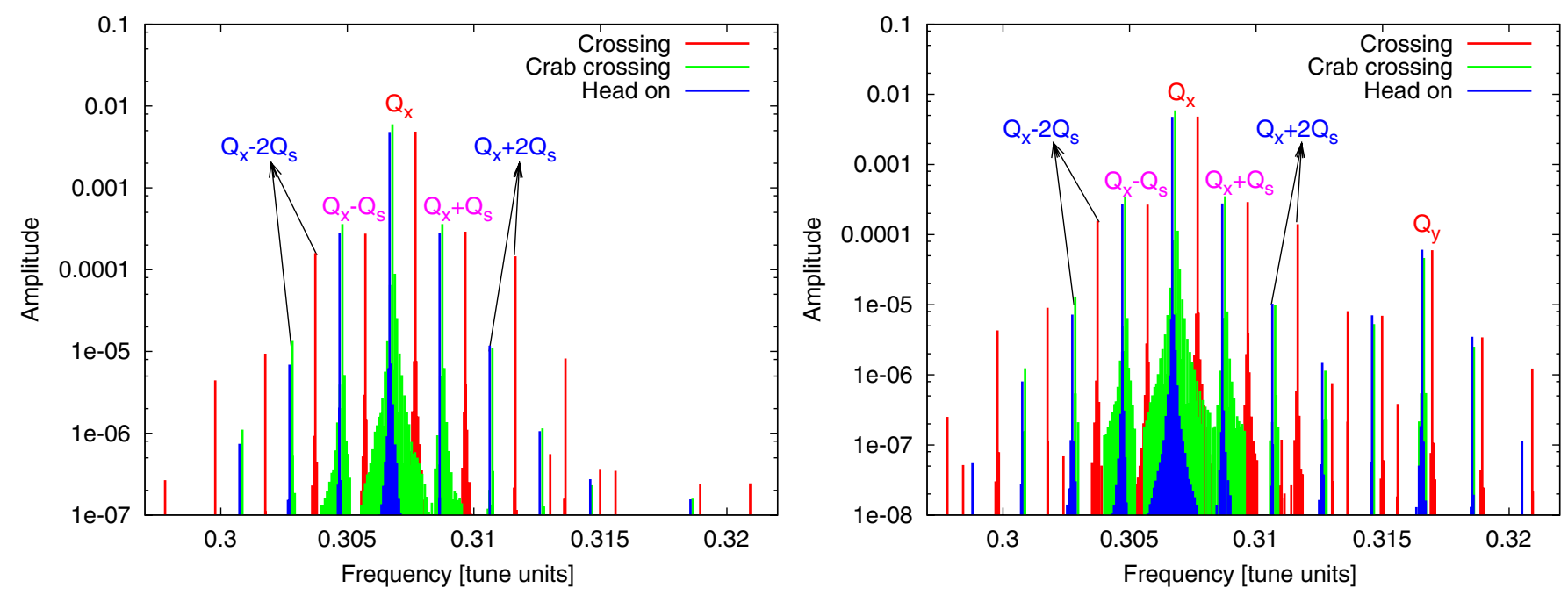

FIG. 29. (Color) Frequency spectrum computed from the turn-by-turn horizontal beam position, without (left) and with (right) nonlinear magnet errors, for a particle launched with $1 \sigma$ offset in $x, y$, and $z$. The second sideband synchrobetatron resonances introduced by the crossing collision are suppressed by the crab cavities (synchrotron tune $Q_{s}=0.00197$, and chromaticity $Q_{x, y}^{\prime}=2$ ). The spectrum with crossing angle and crab cavities resembles the head-on collision case.

crab cavity (crab crossing), and the head-on beam-beam effect without crossing angle (head-on collision). From simulating particle trajectories for these three cases, we computed the fast-Fourier-transform (FFT) of the turn-byturn horizontal position to obtain the spectrum. Particles are tracked over 10000 turns to minimize the influence of noise on the FFT results. Both transverse (horizontal and vertical) initial offsets and the longitudinal initial offset are set to be $1 \sigma$.

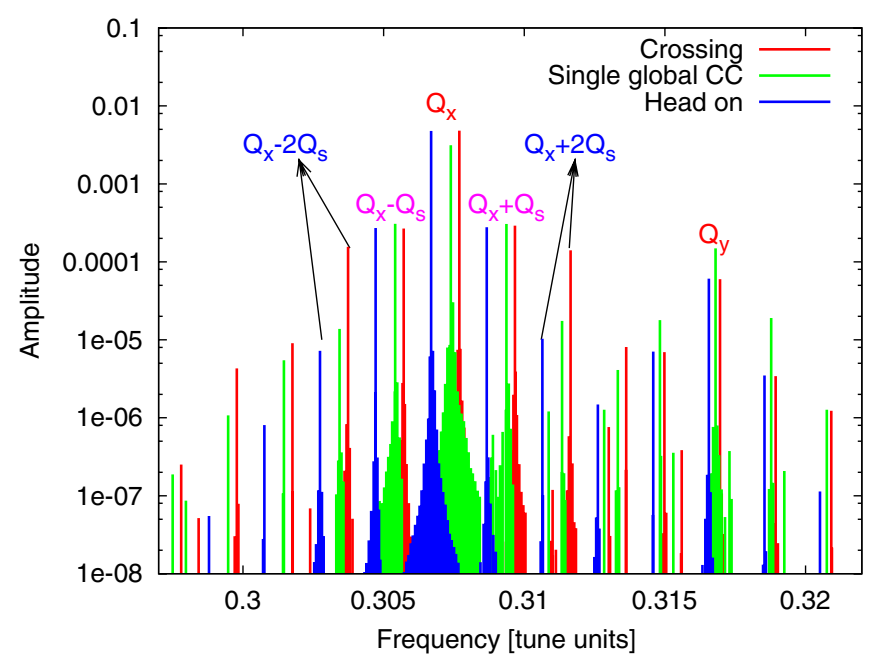

FIG. 30. (Color) Frequency spectrum computed from the turnby-turn horizontal beam position, with nonlinear magnet errors, for a particle launched with $1 \sigma$ offset in $x, y$, and $z$. The second sideband synchrobetatron resonances introduced by the crossing collision are partially suppressed by the single global $800-\mathrm{MHz}$ crab cavity (synchrotron tune $Q_{s}=0.00197$, and chromaticity $\left.Q_{x, y}^{\prime}=2\right)$.
We expect that for the case with beam-beam effects plus crab cavity (crab crossing with two beams crabbed) the synchrobetatron resonance strength is recovered to be similar to the one for the head-on collision case, and that for the crossing collision case the synchrobetatron resonance strength is the largest. Our expectation is confirmed by the results shown in Fig. 29, for simulations performed without [Fig. 29 (left)] and with [Fig. 29 (right)] magnet errors, respectively. The nonlinear magnet errors introduce coupling so that the vertical tune also becomes visible in the (horizontal) spectrum.

In the case of only one global $800-\mathrm{MHz}$ crab cavity (single global scheme with only one beam crabbed) the second-order sidebands due to the crossing angle are only partially compensated by the crab cavity. The frequency spectrum for such a case is shown in Fig. 30, with nonlinear magnet errors included.

\section{LONG-RANGE EFFECTS}

The nominal LHC will operate with 2808 bunches per beam and with a bunch spacing of $25 \mathrm{~ns}$. There are 120 long-range beam-beam interactions in the four interaction regions (IR1, IR2, IR5, and IR8). As additional time slots must be provided for the injection and extraction kickers throughout the LHC complex, bunches at the beginning or end of a bunch train will miss up to half of the long-range beam-beam collisions at one or several IPs (so-called "PACMAN" bunches). For the nominal LHC, the tune shift for PACMAN bunches is less than $5 \times 10^{-4}$ (in units of $2 \pi$ ) [27]. Also the maximum difference in phase advance between the global crab cavity (IR4) and the collision point (IP5) for normal and PACMAN bunches is smaller than $5 \times 10^{-4}$ (in units of $2 \pi$ ). The beta functions, 


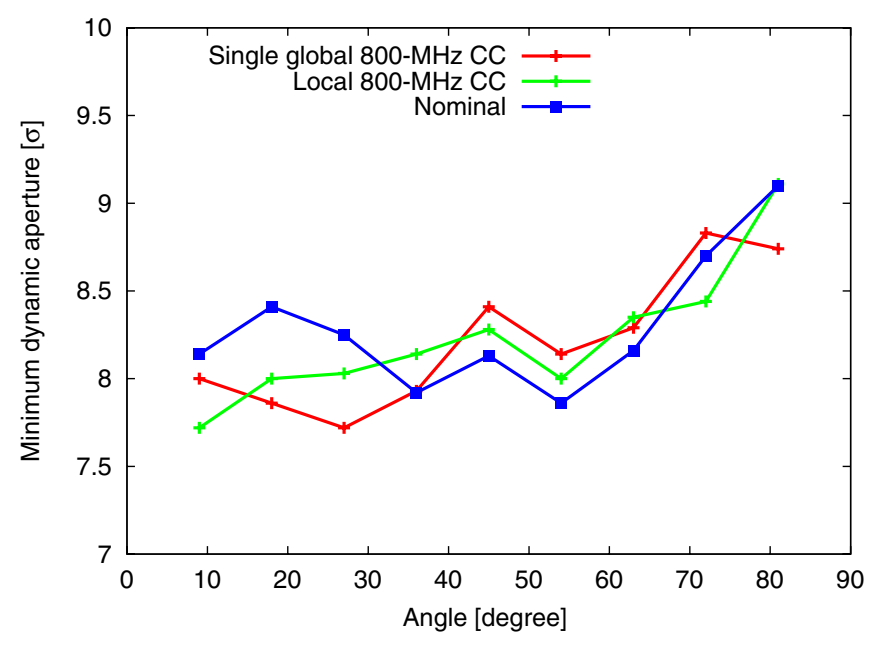

FIG. 31. (Color) Minimum dynamic aperture over 60 error seeds for the nominal LHC optics with or without crab cavity; including all the head-on and long-range beam-beam interactions at IP1, IP2, IP5, and IP8. The dynamic-aperture tracking is performed for 100000 turns.

$\beta_{x}$, at the global CC location and at IP5, are almost unchanged by the missing long-range beam-beam collisions. From this, we conclude that the effect of the longrange collisions on the efficiency of the crab compensation is negligible. The effect of the head-on collision is important, however: For the local crabbing scheme at the nominal LHC, the beam-beam head-on collisions at IP5 and IP1 (plus all long-range collisions) change the horizontal phase advance between the two local crab cavities from 0.517 to 0.510 (in units of $2 \pi$ ), which will, indeed, improve the locality of the crab-cavity $\pi$ bump. The crab-cavity voltage has to be calculated accordingly.

With or without crab cavity, the head-on and especially the long-range beam-beam collisions largely reduce the beam lifetime, which is due to the diffusion of particles with large transverse amplitude. Using the same conventions and simulation recipes as in Sec. IV, the dynamic aperture for the nominal LHC including all the head-on and long-range beam-beam interactions has been calculated both without and with crab cavities. The result is presented in Fig. 31. We observe that in the presence of beam-beam collisions the minimum dynamic aperture over 60 error seeds is around $8 \sigma$ (which is much smaller than the $12.5-13.0 \sigma$ of Figs. 6 and 7), and there is only a small impact from either single global or local crab cavities, as the effect of the long-range collisions is dominant.

\section{CONCLUSIONS}

To increase the luminosity of LHC by a factor of 10 above its design value in the second half of the next decade, several upgrade scenarios have been proposed where crab cavities are an essential ingredient. In this paper, two crab crossing scenarios were studied. The first installs local crab cavities in LHC IR5 (or IR1), to crab the two beams and benefit the proton-proton collision at IP5 (or IP1). The second uses only a single $800-\mathrm{MHz}$ global crab cavity to obtain one horizontally crabbed beam at IP5, in order to test the crab cavity in hadron colliders for the first time.

Various beam dynamics issues have been studied for these two crab crossing schemes (for two LHC optics with two different value of $\beta^{*}$ ), such as optics modifications, dynamic aperture, luminosity, emittance growth from crab cavity ramping up, physical aperture and beta beating, crab crossing tune shift, long-range collisions, and synchrobetatron resonances, crab dispersion, and the impact on the LHC collimation system. In detail, the LHC optics can fulfill the primary requirements for installing local or global crab cavities. The $\beta$ function at the crab cavity $\left(\beta_{\mathrm{CC}}\right)$ can be increased to further decrease the required crab-cavity voltage. For global crab-cavity schemes it is also possible to decrease the crab-cavity voltage by shifting the tune closer to the integer resonance. The minimum dynamic aperture over 60 seeds of the magnetic errors shows a maximum 1 to $2 \sigma$ degradation due to crab cavities, which appears acceptable. The global crabbing scheme for the nominal LHC requires an additional $0.5 \sigma$ aperture from the closed-orbit point of view (while the lowbetamax optics requires more, about $1 \sigma$ ), which should be available, if needed by tighter orbit control. The $z$-dependent "beta beating" due to the global crab cavity is much smaller than the regular off-momentum beta beating. The luminosity gains from crab crossing computed analytically and by GUINEA-PIG simulations show good agreement. For the local crabbing scheme with 400$\mathrm{MHz}$ crab cavities, the luminosity can be recovered so as to be equal to the head-on collision case. For the global crabbing scheme with only one $800-\mathrm{MHz}$ global crab cavity, the luminosity gain can be as large as $25 \%$ for reduced beam emittance and lower bunch intensity. The emittance growth study for the crab-cavity voltage ramping shows that a ramping period longer than 10 turns is needed. An increase in the projected horizontal emittance when the crab cavity is turned on reflects its desired effect of introducing an $x-z$ correlation. The local cleaning inefficiency of the LHC collimation system without errors is not affected by the crab-cavity presence. The available phase space for the circulating beam is only moderately disturbed by the global crab cavity, and, in particular, the hierarchy of primary (TCP), secondary (TCSG), tertiary (TCTH), beam dump (TCDQ) horizontal collimators, and shower absorbers (TCLA) is maintained. For the crab collision case with both beams crabbed, the simulated beam-beam tune shift is found to be the same as the head-on collision tune shift. Simulations demonstrate that second-order synchrobetatron resonances introduced by the crossing collision are suppressed by the crab cavities. The effect of the long-range collisions on the efficiency of the crab compensation is insignificant. In the presence of 
head-on and long-range beam-beam collisions the minimum 100000 -turn dynamic aperture over 60 error seeds is around $8 \sigma$, with or without crab cavities.

In conclusion, all results of our preliminary study support the feasibility of crab-cavity operation in the LHC.

\section{ACKNOWLEDGMENTS}

The authors would like to thank Dr. D. Schulte for providing the simulation code GUINEA-PIG, and Dr. C. Bracco for providing a MATLAB script for collimation studies. The authors would also like to thank Mr. S. White and Dr. J.-P. Koutchouk for helpful discussions, and Dr. M. Giovannozzi, Dr. F. Schmidt, and Dr. U. Dorda for helpful comments and discussions. This work was supported by the European Community-Research Infrastructure Activity under the FP6 "Structuring the European Research Area" program (CARE, Contract No. RII3-CT-2003-506395), and under the FP7 "Capacities Specific Programme" (EuCARD, under Grant Agreement No. 227579).

[1] O. Bruning et al., Report No. CERN 2004-003, 2004.

[2] F. Zimmermann, in Proceedings of the 2007 Particle Accelerator Conference, Albuquerque, New Mexico, 2007 (IEEE, Albuquerque, New Mexico, 2007), p. 714.

[3] R. Calaga et al., in Proceedings of LUMI06, Valencia, Spain, 2006 (Report No. CARE-Conf-07-004-HHH); R. Tomás et al., in Proceedings of LUMI06, Valencia, Spain, 2006 (Report No. CARE-Conf-07-004-HHH); R. Calaga et al., Report No. CARE-HHH-08-002, 2008.

[4] R. Palmer, Report No. SLAC-PUB-4707, 1988.

[5] K. Oide and K. Yokoya, Phys. Rev. A 40, 315 (1989).

[6] J. Irwin, Nucl. Instrum. Methods Phys. Res., Sect. A 298, 460 (1990).
[7] F. Zimmermann and U. Dorda, in Proceedings of LHCLUMI-05 Workshop, Arcidosso, Italy, 2005 (Report No. CARE-Conf-06-014-HHH).

[8] K. Oide et al., in Proceedings of the 2007 Particle Accelerator Conference, Albuquerque, New Mexico, 2007 (Ref. [2]), p. 27.

[9] O. Bruning et al., LHC Project Report No. 1008, 2007.

[10] Y.-P. Sun et al., Report No. CERN-AB-Note-033, 2008; Report No. CERN-BE-Note-019, 2009.

[11] R.D. Maria, presentation on US-LARP CM12 Collaboration Meeting, 2009.

[12] M. Aiba et al., in Proceedings of the 11th European Particle Accelerator Conference, Genoa, 2008 (EPS-AG, Genoa, Italy, 2008), p. 2524.

[13] F. Schmidt, Report No. CERN SL/94-56 (AP), 1994.

[14] LHC Design Report Volume I, The LHC main ring, CERN, 2004, p. 86.

[15] D. Schulte, Ph.D. thesis, University Hamburg, 1996.

[16] R. Assmann et al., LHC Project Document No. LHC-BES-0007, 2004, https://edms.cern.ch/file/347396/1.1/ LHC-B-ES-0007-10-00.pdf.

[17] A. Morita, CERN Report No. BE-Note-2009-007, 2009.

[18] MADX manual, http://mad.web.cern.ch/mad/.

[19] Y.-P. Sun et al., Report No. CERN-BE-2009-012, 2009.

[20] R. Assmann et al., LHC Project Note 277, 2002.

[21] C. Bracco, Ph.D. thesis, Ecole Polytechnique Federale de Lausanne, Lausanne, Switzerland [Report No. CERNTHESIS-2009-031, 2009].

[22] K. Hirata, Phys. Rev. Lett. 74, 2228 (1995).

[23] L. Leunissen, F. Schmidt, and G. Ripken, Phys. Rev. ST Accel. Beams 3, 124002 (2000).

[24] K. Hirata, H. Moshammer, and F. Ruggiero, Part. Accel. 40, 205 (1993).

[25] Y.-P. Sun, R. Tomás, and F. Zimmermann, in Proceedings of Particle Accelerator Conference 2009 (IEEE, Vancouver, Canada, 2009), TH5PFP016.

[26] A. Piwinski, IEEE Trans. Nucl. Sci. 24, 1480 (1977).

[27] F. Zimmermann, in Proceedings of the 9th European Particle Accelerator Conference, Lucerne, 2004 (EPSAG, Lucerne, 2004), p. 83. 